\title{
Cost-Sensitive Learning of Fuzzy Rules for Imbalanced Classification Problems Using FURIA
}

\author{
Ana Palacios \\ Dpto. de Ciencias de la Computación e I. A., Univ. de Granada, Spain
}

Krzysztof Trawiński

European Centre for Soft Computing, Asturias, Spain

Oscar Cordón

Dpto. de Ciencias de la Computación e I. A. and CITIC-UGR, Univ. de Granada, Spain European Centre for Soft Computing, Asturias, Spain

\author{
Luciano Sánchez \\ Dpto. de Informática, Univ. de Oviedo, Spain \\ luciano@uniovi.es
}

Received 17 March 2013

Revised 26 May 2014

\begin{abstract}
This paper is intended to verify that cost-sensitive learning is a competitive approach for learning fuzzy rules in certain imbalanced classification problems. It will be shown that there exist cost matrices whose use in combination with a suitable classifier allows for improving the results of some popular data-level techniques. The well known FURIA algorithm is extended to take advantage of this definition. A numerical study is carried out to compare the proposed cost-sensitive FURIA to other state-of-the-art classification algorithms, based on fuzzy rules and on other classical machine learning methods, on 64 different imbalanced datasets.
\end{abstract}

Keywords: Fuzzy rule learning; imbalanced datasets; cost-sensitive learning; FURIA; SMOTE.

\section{Introduction}

The problem of imbalanced datasets in classification or "datasets with rare classes" occurs when the number of instances of a class is much lower than that of the other classes. ${ }^{49}$ In these problems it often happens that the minority class is the most interesting. However, minimum-error oriented classifiers tend to ignore the minority class and produce wrong conclusions. ${ }^{17,29,38,40,49}$ This happens in many applications such as medical diagnosis, ${ }^{35}$ fraud detection, ${ }^{39}$ risk management, ${ }^{24}$ among others.

Solving the imbalanced learning problem consists of reducing the false negatives as much as possible without increasing too much the number of false 
positives. The strategies for achieving this objective can be grouped into two principal categories: ${ }^{11}$ cost-sensitive learning or internal approach and data-level or external approach. For internal methods, classifiers optimizing criteria different than the expected error rate are sought. For example, the minimum risk Bayes rule ${ }^{4}$ is implicit or explicitly adopted in certain methods ${ }^{13,14,17,53}$ where a higher risk (proportional to the imbalance ratio, i.e., to the ratio between the a priori probabilities for the minority class and the remaining classes) is assigned to misclassifications in the minority class. In contrast, in external methods, data is preprocessed for equalizing the prior probabilities of the classes. Oversampling, undersampling or combinations of both are used for rebalancing false positives and negatives. ${ }^{3,5,44}$

Other authors ${ }^{37}$ suggest that for every performance criteria, for example area under the ROC curve, ${ }^{10,23}$ or arithmetic or geometric mean of the confusion matrix diagonal, ${ }^{31}$ a cost matrix can be found for which the optimal classifier coincides with the minimum risk Bayes rule. However, the method for computing this cost matrix is still undefined. Lastly, there are not many publications detailing numerical experimentations where the performance of both internal and external approaches are compared. It is worth mentioning that some authors claim that cost-sensitive learning does not improve preprocessing algorithms, albeit the differences found in these studies were not statistically significant. ${ }^{32}$

Multiple studies regarding fuzzy rule-based classification systems (FRBCSs) have been published. Learning fuzzy rules or fuzzy decision trees from imbalanced datasets has been solved with scalar $9,32,36,42,47,48,51$ and multi-objective techniques. ${ }^{16,19}$ In particular, imbalanced classification has been regarded as a multiobjective problem, where accuracy and complexity are balanced and the ROC convex hull used to select a good trade-off. ${ }^{16}$ An external approach has also been shown to produce good results. ${ }^{18,20-22}$ In the current contribution it will be shown that cost-sensitive learning can be at least as effective or even better than preprocessing the data. For this purpose, the Fuzzy Unordered Rule Induction Algorithm (FURIA $)^{25,27}$ will be generalized to cost-sensitive learning. In addition, two heuristics are proposed for defining the cost matrix in terms of the classification problem imbalance ratio. The results of this new algorithm, that will be called FURIA costsensitive (FURIA_CS), will be compared to those of FURIA on datasets that have been rebalanced with state-of-the-art methods, including Synthetic Minority Oversampling Technique (SMOTE) ${ }^{5}$ and its variant with the Wilson's Edited Nearest Neighbor rule (ENN). ${ }^{52}$ These techniques have been chosen because of their robust behaviours. ${ }^{3,18}$

This paper is organized as follows. Section 2 introduces the problem of imbalanced datasets. Preprocessing methods, cost-sensitive learning, and the employed metrics are defined in this part. Section 3 recalls the parts of the FURIA algorithm relevant to this study. Section 4 introduces FURIA_CS and makes a detailed description of the effected changes. In Sec. 5, numerical results are provided. FURIA_CS is compared to a combination of FURIA with preprocessing and to other selected state-of-the-art classification algorithms. The paper concludes in Sec. 6. 
Table 1. Confusion matrix for two classes problems.

\begin{tabular}{l|c|c}
\hline & Positive class & Negative class \\
\hline Positive Prediction & True Positive (TP) & False Positive (FP) \\
Negative Prediction & False Negative (FN) & True Negative (TN) \\
\hline
\end{tabular}

\section{Imbalanced Classification Problem. Notation and Metrics for Two-Classes Problem}

In two-classes problems, the confusion matrix divides the results of classifying a set of instances into four different categories, as shown in Table 1: true positive (TP), true negative $(\mathrm{TN})$, false positive $(\mathrm{FP})$ and false negative $(\mathrm{FN})$.

The fraction of misclassified instances is

$$
\operatorname{Err}=\frac{\mathrm{FP}+\mathrm{FN}}{\mathrm{TP}+\mathrm{TN}+\mathrm{FP}+\mathrm{FN}}
$$

while the accuracy is 1-Err.

For independently measuring the classification quality for positive and negative classes, the following values are defined:

$$
\begin{array}{ll}
\mathrm{TP}_{\text {rate }}=\frac{\mathrm{TP}}{\mathrm{TP}+\mathrm{FN}}, & \mathrm{FN}_{\text {rate }}=\frac{\mathrm{FN}}{\mathrm{TP}+\mathrm{FN}} \\
\mathrm{TN}_{\text {rate }}=\frac{\mathrm{TN}}{\mathrm{TN}+\mathrm{FP}}, & \mathrm{FP}_{\text {rate }}=\frac{\mathrm{FP}}{\mathrm{TN}+\mathrm{FP}}
\end{array}
$$

and the terms "specificity" or acc $=\mathrm{TN}_{\text {rate }}$, and "sensitivity" or acc ${ }^{+}=\mathrm{TP}_{\text {rate }}$ are commonly used.

Learning algorithms minimizing the fraction of misclassified instances tend to produce classifiers where $\mathrm{TN}_{\text {rate }}$ is too low. ${ }^{40,49}$ For this reason, criteria more appropiate than the average classification error are considered. ${ }^{31}$ The most common metrics for imbalanced, two-classes problems are:

- The geometric mean $(\mathrm{GM})^{31}$ of the sensitivity and the specificity. GM is an interesting indicator of the quality of a classifier for imbalanced data, because it is high when both $\mathrm{acc}^{+}$and acc are high or when the different between acc ${ }^{+}$ and acc is small. ${ }^{30}$

- The Area Under the ROC Curve (AUC) ${ }^{10,23}$ which is a trade-off between benefits $\left(\mathrm{TP}_{\text {rate }}\right)$ and costs $\left(\mathrm{FP}_{\text {rate }}\right)$. AUC is approximated by the value that follows:

$$
\mathrm{AUC}=\frac{1+\mathrm{TP}_{\text {rate }}-\mathrm{FP}_{\text {rate }}}{2}
$$

As already mentioned, there are two approaches for solving imbalanced classification problems: cost-sensitive learning and preprocessing for equalizing the prior probabilities of the classes. Both will be described in the following sections. 


\subsection{Cost-sensitive learning}

Cost-sensitive learning ${ }^{14,17}$ can be categorized into two classes: ${ }^{45}$

- Class-dependent costs. ${ }^{14,17,50,55}$ The cost depends on the pair (true class, assigned class).

- Example-dependent costs. 1,33,34,53,54 Different examples can have different misclassification costs, irrespectively of their true classes or the classes they are assigned.

In this paper, classifiers of the first category are used. These classifiers depend on a cost matrix $C$, where $C(i, j)$ is the cost of assigning the class $i$ to an example whose true class is $j$. In binary classification problems, the notation $C(+,-)$ is used for naming the cost of misclassifying a positive (minority class) example, and $C(-,+)$ is the cost of the opposite case. It is needed that the cost of misclassifying instances of the minority class is higher or equal than the cost of misclassifying the majority class, i.e. $C(+,-) \geq C(-,+)$. It is intuitive, but not mandatory that $C(-,-)=C(+,+)=0 .{ }^{17,45}$ Heuristic cost assignments are common. ${ }^{43,44}$

\subsection{Preprocessing imbalanced datasets. SMOTE and SMOTE + ENN algorithms}

In this paper, the SMOTE algorithm, ${ }^{5}$ and a hybrid approach, $\mathrm{SMOTE}+\mathrm{ENN}^{3}$ are used. In the SMOTE algorithm, the minority class is over-sampled. New synthetic instances are introduced along the line segments joining any or all of the nearest neighbors of each instance in the minority class. SMOTE + ENN is a variant of SMOTE where Wilson's ENN Rule ${ }^{52}$ is used after oversampling for removing from the training set any example whose class is not in agreement with its three nearest neighbours.

\section{FURIA Outline}

Fuzzy Unordered Rules Induction Algorithm (FURIA) ${ }^{25,27}$ is a novel fuzzy rulebased classification method extending the classical RIPPER. ${ }^{7}$ The most important differences between FURIA and RIPPER concern the type of of rule model and the use of default rules. ${ }^{27}$

With respect to the rule model type, FURIA performs a fuzzification of the rule antecedents, using a greedy algorithm that extends the support of each rule so as to improve a purity criteria measuring the component-wise confidence of the fuzzy classification rule. With respect to the use of default decisions, rules in RIPPER are in ascending order by the prior probability of the classes in their consequents. The first rule matching the query pattern is used for classifying it. Uncovered examples are assigned to the most frequent class (default rule). In contrast, FURIA uses a one-vs-rest decomposition. No default rule is needed and the order of the classes is irrelevant, but uncovered instances may happen. When a query instance 
is uncovered by the fuzzy classification rules derived from FURIA, the nearest rule in the fuzzy knowledge base is applied to the query. This fuzzy rule is determined by a process called "rule stretching", where all rules are gradually generalized until one of the stretched antecedents is satisfied by the uncovered instance.

In order to make this paper more self-contained, an algorithmic description of FURIA is included below, where the parts that will be altered in the cost-based learning generalization (see Sec. 4) are marked in boldface. The interested reader is referred to the original references ${ }^{25,27}$ and also to the source code of the software implementation provided by the authors ${ }^{26}$ for a full description of FURIA.

The outer loop of the FURIA algorithm is as follows:

\section{Method FURIA()}

Select a class and learn crisp classification rules discriminating this class from the others (call method RuleSetForOneClass())

Remove redundant antecedents

Fuzzify rules maximizing the purity of the fuzzification of each attributte Compute confidence degrees for all rules considering the certainty factor

Evaluate rules and apply rule stretching if there are uncovered examples End of Method

This schema needs not to be altered in order to introduce classification costs, however there are three parts that need a new, cost-based definition:

(1) the rule purity, that quantifies the quality of the fuzzification procedure, depends on the costs of the partially covered examples

(2) the certainty factor, that measures the confidence assigned to the piece of information described by the rule, depends also on the costs

(3) the rule stretching procedure, that is used to simplify the antecedents for improving generalization, should not depend on the number of examples covered by the rule but on their relative costs.

Second, the method RuleSetForOneClass () referenced before, contains a pruning stage that depends on the cost matrix too. The pseudocode of this method is as follows:

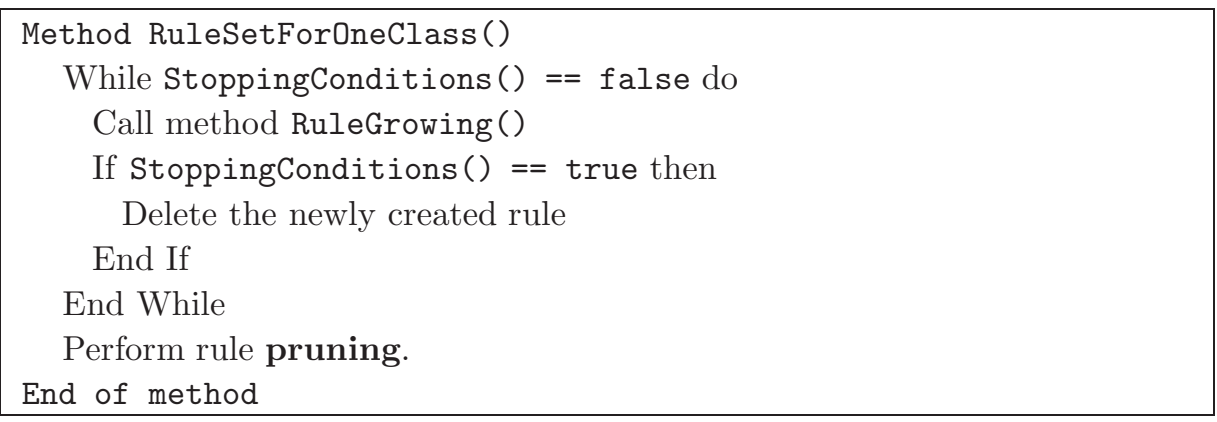


Third, the method RuleGrowing() is based on a measure of information gain. The information gain in error-based classification depends on the probabilities of the classes, nonetheless probabilities must be replaced by expected costs in this context. The pseudocode of this method follows:

Method RuleGrowing()

Grow rule using an information gain measure to choose the best conjunct to be added into the rule antecedent.

Stop adding conjuncts when the rule starts covering negative instances.

End of method

Lastly, the stopping conditions of FURIA are based on classification error and that must be updated to classification risk. These conditions are:

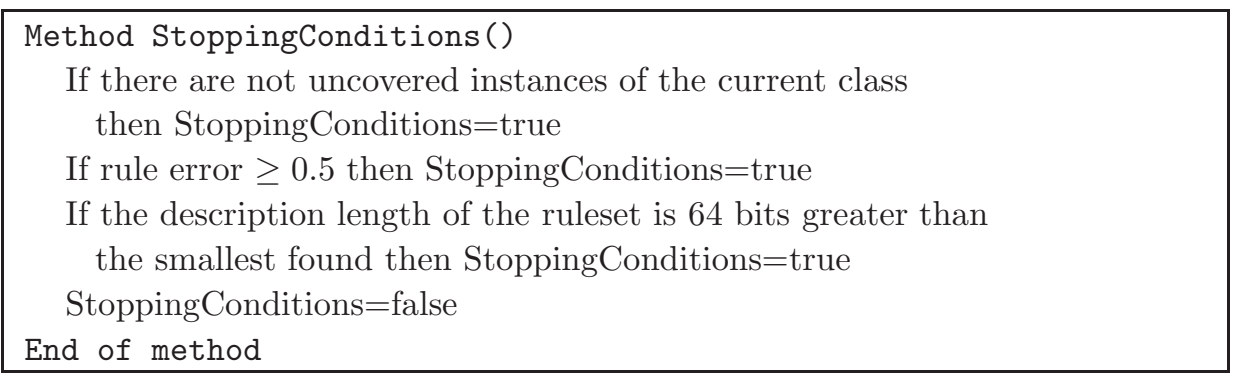

\section{A Proposal for a Cost-Sensitive FURIA Algorithm}

Those parts marked in boldface in the preceding description will be explained in detail in this section, along with their proposed extensions to cost-based classification. In the following, the training set is $D \subset \mathbb{R}^{k}$ and instances are vectors $x=\left(x_{1}, \ldots, x_{k}\right) \in D$. Each antecedent of a FURIA fuzzy classification rule is a multivariate trapezoidal fuzzy set whose membership is

$$
I^{F}(x)=\bigoplus_{i=1, \ldots k} I_{i}^{F}\left(x_{i}\right)
$$

and its core is the interval $I=I_{1} \times \cdots \times I_{k}$, where the indicator function of $I_{i}$, $i=1, \ldots, k$ is

$$
I_{i}\left(x_{i}\right)= \begin{cases}1 & \text { if } I_{i}^{F}\left(x_{i}\right)=1 \\ 0 & \text { else }\end{cases}
$$

and the operator $\oplus$ is the fuzzy addition,

$$
\mu_{A \oplus B}(x)=\sup _{a+b=x}\left\{\alpha \mid \min \left(\mu_{A}(a), \mu_{B}(b)\right) \geq \alpha\right\} .
$$

\subsection{Information gain}

This criterion measures the improvement of a rule with respect to the default for the target class and is used as a stopping condition in the rule growing procedure. 
Let $I$ be the core of the antecedent of the rule at hand, and let $l$ be the target class. Then, the number of positive examples for the fuzzy classification rule $r$ is

$$
p_{r}=\#\{x \in I \mid \operatorname{class}(x)=l\}
$$

and the number of negative examples for that rule is

$$
n_{r}=\#\{x \in I \mid \operatorname{class}(x) \neq l\} .
$$

The total number of positive and negative examples in the dataset are named $p$ and $n$, respectively. Then, the information gain is defined as follows: ${ }^{26}$

$$
\mathrm{IG}_{r}=p_{r} \times\left(\log _{2}\left(\frac{p_{r}+1}{p_{r}+n_{r}+1}\right)-\log _{2}\left(\frac{p+1}{p+n+1}\right)\right) .
$$

The information gain depends on the quotient between the expected fraction of instances well classified by the rule at hand and by the default rule, as well as the fraction of the number of positive examples for the rule $r$ and by the number of negative examples for that rule $r$. These expressions must guard against the division by zero, thus the approximations

$$
\frac{p}{p+n} \approx \frac{p+1}{p+n+1}
$$

and

$$
\frac{p_{r}}{p_{r}+n_{r}} \approx \frac{p_{r}+1}{p_{r}+n_{r}+1}
$$

were made in the reference software implementation of FURIA. ${ }^{26}$

\subsubsection{Cost-sensitive extension}

The proposed generalization of this expression to cost-based learning consists of replacing the expected fraction of misclassified instances by the expected risk.

Let $l$ be the class in the consequent of the rule being grown and $I$ the core or its antecedent, then the cost-sensitive version of the number of positive examples $p_{r}$ is defined as follows:

$$
p_{r}^{\mathrm{CS}}=\sum_{x \in I} 1-C(l, \operatorname{class}(x)) .
$$

Notice that, if the cost of every misclassification was 1 ,

$$
C(i, j)= \begin{cases}0 & \text { if } i=j \\ 1 & \text { if } i \neq j\end{cases}
$$

then $p_{r}^{\mathrm{CS}}=p_{r}$, the number of positive examples for the rule at hand. Analogously, the number of positive instances in the dataset is generalized to

$$
p^{\mathrm{CS}}=\sum_{x \in D} 1-C(l, \operatorname{class}(x)) .
$$


Hence, the cost-based information gain is defined as follows:

$$
\mathrm{IG}_{r}^{\mathrm{CS}}=p_{r}^{\mathrm{CS}} \times\left(\log _{2}\left(\frac{p_{r}^{\mathrm{CS}}+1}{p_{r}+n_{r}+1}\right)-\log _{2}\left(\frac{p^{\mathrm{CS}}+1}{p+n+1}\right)\right) .
$$

\subsection{Pruning}

Each rule comprises $q$ antecedents, that will be named $a_{1}, \ldots, a_{q}$. The list $\left\langle a_{1}, \ldots, a_{q}\right\rangle$ makes reference to an AND combination of these antecedents. Antecedents comprise three parts:

- The index of an attribute

- The split point of this attribute

- The condition for comparing the value of the attribute and the split point (lower or equal, higher or equal).

For instance, the antecedent $(2,3, \leq)$ is true if the value of the second variable is lower or equal than 3 .

The order of the antecedents reflects their importance thus pruning a rule consists of selecting a sublist $\left\langle a_{1}, \ldots, a_{i}\right\rangle$, with $i \leq q$. In order to find a suitable value for $i$, the following rule-value metric is computed first: ${ }^{26}$

$$
V_{r}=\frac{p_{r}+1}{p_{r}+n_{r}+2} .
$$

Let the number of positive covered and negative uncovered examples of the rule, when pruned at the $i$-th antecedent, respectively be $P_{i}$ and $N_{i}$ :

$$
\begin{aligned}
P_{i} & =\#\left\{x \mid x \text { is covered by }\left\langle a_{1}, \ldots, a_{i}\right\rangle \wedge \operatorname{class}(x)=l\right\}, \\
N_{i} & =\#\left\{x \mid x \text { is not covered by }\left\langle a_{1}, \ldots, a_{i}\right\rangle \wedge \operatorname{class}(x) \neq l\right\} .
\end{aligned}
$$

and let be defined the value ${ }^{26}$

$$
\text { worth }_{i}=\frac{P_{i}+N_{i}}{p+n} .
$$

This value measures how likely is each antecedent to be pruned. If

$$
\max _{i=1, \ldots, q} \operatorname{worth}_{i}>V_{r}
$$

then the term where the value of " worth $_{i}$ " is maximum is selected for pruning.

\subsubsection{Cost-sensitive extension}

The extension of the value defined in Eq. (16) is

$$
V_{r}^{\mathrm{CS}}=\frac{p_{r}^{\mathrm{CS}}+1}{p_{r}+n_{r}+2} .
$$

In addition, the worth concept is also extended as follows:

$$
\operatorname{worth}_{i}^{\mathrm{CS}}=\frac{P_{i}^{\mathrm{CS}}+N_{i}^{\mathrm{CS}}}{p+n}
$$


Table 2. Dataset for example 1.

\begin{tabular}{c|c|c|c|c}
\hline Instance & $x_{0}$ & $x_{1}$ & $x_{2}$ & Class \\
\hline 1 & 1 & 1 & 3 & 1 \\
2 & 1 & 2 & 4 & $\mathbf{2}$ \\
3 & 2 & 2 & 4 & 1 \\
4 & 1 & 1 & 2 & 1 \\
5 & 3 & 3 & 3 & 1 \\
6 & 3 & 4 & 3 & 1 \\
\hline
\end{tabular}

where

$$
\begin{aligned}
P_{i}^{\mathrm{CS}} & =\sum_{\left\{x \text { is covered by }\left\langle a_{1} \cdots a_{i}\right\rangle\right\}} 1-C(l, \operatorname{class}(x)), \\
N_{i}^{\mathrm{CS}} & =\sum_{\left\{x \text { is not covered by }\left\langle a_{1} \cdots a_{i}\right\rangle\right\}} C(l, \operatorname{class}(x)) .
\end{aligned}
$$

The following example clarifies the meaning of this generalized pruning in an imbalanced classification context.

Example 1. Let $D \subset \mathbb{R}^{3}$ be the dataset in Table 2, comprising 6 instances of classes 1 (majority) and 2 (minority). The list of antecedents of the rule to be pruned is $\left\langle a_{1}, a_{2}\right\rangle$. For example, $a_{1}$ and $a_{2}$ are as follows:

$$
\begin{aligned}
& a_{1}=(1,2, \leq), \\
& a_{2}=(2,2, \leq) .
\end{aligned}
$$

The consequent of the rule is "class is 2 ". Instances \#1,\#2,\#3 and \#4 are compatible with $a_{1}$. Instance \#4 is compatible with both $a_{1}$ and $a_{2}$.

Applying Eq. (19), the following results are obtained:

$$
\begin{aligned}
& \text { worth }_{1}=\frac{P_{1}+N_{1}}{p+n}=\frac{1+2}{6}=0.5, \\
& \text { worth }_{2}=\frac{P_{2}+N_{2}}{p+n}=\frac{0+4}{6}=0.66 .
\end{aligned}
$$

Since worth is greater than worth $_{1}$, the cut point is $i=2$ and therefore the rule is not pruned.

Suppose that the following cost matrix is adopted:

\begin{tabular}{l|c|c}
\hline & Positive class & Negative class \\
\hline Positive Prediction & 0 & 0.25 \\
\hline Negative Prediction & 1 & 0 \\
\hline
\end{tabular}


Applying Eq. (22), the results are:

$$
\begin{aligned}
& \operatorname{worth}_{1}^{\mathrm{CS}}=\frac{P_{1}^{\mathrm{CS}}+N_{1}^{\mathrm{CS}}}{p+n}=\frac{3.25+0.5}{6}=0.625, \\
& \text { worth }_{2}^{\mathrm{CS}}=\frac{P_{2}^{\mathrm{CS}}+N_{2}^{\mathrm{CS}}}{p+n}=\frac{0.75+1}{6}=0.29 .
\end{aligned}
$$

In this case, worth ${ }_{1}^{C S}$ is greater than worth ${ }_{2}^{C S}$ and the rule is pruned at $i=1$. The higher cost assigned to the misclassification of the minority class ( 4 times higher than the opposite) produces a pruning where the simplified rule covers instance \#2, the element of the minority class in the dataset. By contrast, instance \#2 was not covered by the pruned rule if an error-based approach was followed, as seen in the first part of this example.

\subsection{Purity}

This value measures the quality of the fuzzification procedure and it is used for determining the support of the fuzzy sets defining the rule antecedents. Let $D^{i}$ be the subset of the training data that follows:

$$
D^{i}=\left\{\left(x_{1}, \ldots, x_{k}\right) \mid x_{j} \in I_{j}^{F}\left(x_{j}\right) \text { for all } j \neq i\right\} .
$$

$D$ is partitioned into positive and negative instances, $D_{+}^{i}$ and $D_{-}^{i}$. Given the values

$$
\begin{aligned}
p_{i} & =\sum_{x \in D_{+}^{i}} I_{i}^{F}\left(x_{i}\right), \\
n_{i} & =\sum_{x \in D_{-}^{i}} I_{i}^{F}\left(x_{i}\right),
\end{aligned}
$$

the purity of the fuzzification of the $i$-th attribute is: ${ }^{26}$

$$
\operatorname{pur}_{r}=\frac{p_{i}}{p_{i}+n_{i}} .
$$

\subsubsection{Cost-sensitive extension}

The extension of Eq. (34) to cost-sensitive learning is

$$
\operatorname{pur}_{r}^{\mathrm{CS}}=\frac{p_{i}^{\mathrm{CS}}}{p_{i}+n_{i}}
$$

where

$$
p_{i}^{\mathrm{CS}}=\sum_{x \in D^{i}} I_{i}^{F}\left(x_{i}\right)(1-C(l, \operatorname{class}(x)))
$$

and $p_{i}, n_{i}$, were defined in Eqs. (32) and (33). 


\subsection{Certainty factor}

The certainty factor $\mathrm{CF}$ of a rule $\left\langle I^{F}, l\right\rangle$, for a training set $D_{T}$, is: ${ }^{26}$

$$
\mathrm{CF}=\frac{2 \cdot \frac{\sum_{x \in D_{T}, \operatorname{class}(x)=l} p(x)}{\sum_{x \in D_{T}} p(x)}+\sum_{x \in D_{T}, \operatorname{class}(x)=l} I^{F}(x)}{2+\sum_{x \in D_{T}} I^{F}(x)}
$$

where $p(x)$ is the weight of instance $x$, often 1 . It is remarked that the FURIA algorithm is able to learn from a weighed dataset where the contribution of each instance to the total classification error is a preset value, however these weights $p(x)$ are not related to the cost matrix neither they evolve during the learning process.

\subsubsection{Cost-sensitive extension}

The cost-sensitive certainty factor of a rule $\left\langle I^{F}, l\right\rangle$, for a training set $D_{T}$, is:

$$
\mathrm{CF}^{\mathrm{CS}}=\frac{2 \cdot \overline{\mathrm{acc}}^{\mathrm{CS}}+\sum_{x \in D_{T}} I^{F}(x)(1-C(l, \operatorname{class}(x))}{2+\sum_{x \in D_{T}} I^{F}(x)}
$$

where

$$
\overline{\operatorname{acc}}^{\mathrm{CS}}=\frac{\sum_{x \in D_{T}} p(x)(1-C(l, \operatorname{class}(x)))}{\sum_{x \in D_{T}} p(x)}
$$

and $p(x)$ is the weight of instance $x$, mentioned before.

\subsection{Rule stretching}

Rule stretching (or generalization) deals with uncovered examples (those classified by the default rule in RIPPER). The generalization procedure consists of making (preferably minimal) simplifications of the antecedents of the rules until the query instance is covered. The instance is then classified by the rule with the highest evaluation, according to the value ${ }^{26}$

$$
\mathrm{STR}=\mathrm{CF} \cdot \frac{k+1}{m+2} \cdot I^{F}(x)
$$

where $k$ is the size of the generalized antecedent and $m$ is the size of the entire antecedent before applying this procedure. Notice that, $\frac{k+1}{m+2}$ aims at discarding heavily pruned rules. If no streched rule is able to cover the given example $x_{i}$, it is assigned a class based on the a priori distribution. 


\subsubsection{Cost-sensitive extension}

The cost-sensitive extension of Eq. (40) is straightforward:

$$
\operatorname{STR}^{\mathrm{CS}}=\mathrm{CF}^{\mathrm{CS}} \cdot \frac{k+1}{m+2} \cdot I^{F}(x)(1-C(l, \operatorname{class}(x))) .
$$

If the query example cannot be covered by any stretched rule, the class with minimum a priori risk is chosen. The risk of a class $\lambda=1, \ldots, q$ is estimated as follows:

$$
\operatorname{risk}(\lambda)=\sum_{x \in D_{T}} C(\lambda, \operatorname{class}(x))
$$

\subsection{Stopping conditions}

The three following are considered:

(1) There are no more uncovered positive examples in the dataset.

(2) The description length of the ruleset is 64 bits greater than the smallest value met so far.

(3) The number of false positives of a rule, divided by the number of covered instances, is greater or equal than 0.5. In other words, the error rate of the rule is greater or equal than 0.5 .

In this section, a cost-sensitive adaptation of FURIA, named FURIA_CS is described. This adaptation is designed for tackling imbalanced classification problems. The extended algorithm depends on a cost matrix $C$, where $C(i, j)$ is the cost of assigning the $i$-th class to an example whose true class is $j$, as discussed in Sec 2.1. Without loss of generality, it will be assumed that $C(i, j) \leq 1$ for all $i, j$.

The expressions used in the preceding section for computing information gain, pruning, purity, certainty factor and rule stretching, as well as the stopping conditions, must be adapted to reflect these costs, as described in the paragraphs that follow.

\subsubsection{Cost-sensitive extension}

The algorithm proposed here must be stopped when the risk of the rule is higher than certain threshold relative to the maximum risk. It is proposed that the learning will be ended when the error rate of the rule surpasses the STC values defined below, which are based on the imbalance ratio (IR) of the current one vs. others classification problem:

- If the consequent of the rule is the majority class,

$$
\mathrm{STC}_{\mathrm{maj}}= \begin{cases}\frac{1}{\mathrm{IR}} & \text { if IR }>2 \\ 0.5 & \text { else. }\end{cases}
$$


- If the consequent of the rule is the minority class,

$$
\mathrm{STC}_{\min }= \begin{cases}1-\frac{1}{\mathrm{IR}} & \text { if IR }>2 \\ 0.5 & \text { else. }\end{cases}
$$

\section{Experimental Study}

The purpose of the experimental study is to show that cost-sensitive algorithms are competitive against state-of-the-art preprocessing algorithms when learning fuzzy rules for imbalanced classification problems. The experimental setup, comprising a description of datasets, data partitions, selected classifiers of different types, parameters of the classifiers, and misclassification costs is described in Sec. 5.1. In Sec. 5.2, the performance of FURIA_CS, FURIA+SMOTE and FURIA+SMOTE_ENN is compared for two different misclassification costs and cost matrices. Lastly, in Sec. 5.3, FURIA_CS is compared to the results of several classifiers of different types: C4.5, ${ }^{41} \mathrm{SVM}^{46}{ }^{4} \mathrm{FH}-\mathrm{GBML}^{28}$ and $\mathrm{k}-\mathrm{NN} .{ }^{8}$

\subsection{Experimental setup: Datasets, data partitions and parameters}

Sixty-four binary classification problems from the KEEL dataset repository ${ }^{2}$ were selected. The imbalance ratio of all of them is higher than 1.8 and the datasets are divided into three categories: low (IR $<9)$, medium $(9 \leq \mathrm{IR}<11)$ and high (IR $\geq 11$ ). Their properties are summarized in Table 3, where "\#Ex." represents the number of examples, "\#Atts." the number of attributes, "Class $(-,+)$ " the name of each class, "\%Class $(-,+)$ " the percentage of each class, and "IR" the class distribution (i.e., the imbalance ratio). The outcomes of the application of SMOTE and SMOTE+ENN to these datasets have also been obtained from the same data repository.

The experimental design follows a 5-fold cross validation model (5-cv): 5 random partitions of data, $20 \%$ for testing. The error values in this section are the average test results at these 5 partitions.

FURIA_CS depends on a cost matrix (see Table 4). Cost tables are normalized ${ }^{17}$ and the cost of misclassifying a positive example is $\mathrm{C}(+,-)=1 / \mathrm{IR}$ while the cost of misclassifying a negative example is $\mathrm{C}(-,+)=1$. A penalization factor $\mathrm{PF}$ will be assigned to each correct classification of a negative example. ${ }^{45}$

Four classifiers of different types will be considered to benchmark the performance of FURIA_CS: the classical C4.5 method to derive decision trees; ${ }^{41}$ Support Vector Machine (SVM) implementation; ${ }^{46}$ K-nearest Neighbor $(\mathrm{K}-\mathrm{NN}) ;^{8}$ and a state-of-the-art FRBCS learning method, Fuzzy Hybrid Genetic-based Machine Learning (FH-GBML). ${ }^{28}$ Parameters defining these four classifiers are shown in Table 5 and were selected to match those in previous references. ${ }^{32}$ 
Table 3. Summary of the imbalanced datasets.

\begin{tabular}{|c|c|c|c|c|c|}
\hline Data-sets & \#Ex. & \#Atts. & Class $(-,+)$ & \%Class $(-,+)$ & IR \\
\hline Glass1 & 214 & 9 & (build-win-non float-proc; remainder) & $(35.51,64.49)$ & 1.82 \\
\hline Ecoli0vs1 & 220 & 7 & $(\mathrm{im} ; \mathrm{cp})$ & $(35.00,65.00)$ & 1.86 \\
\hline Wisconsin & 683 & 9 & (malignant; benign) & $(35.00,65.00)$ & 1.86 \\
\hline Pima & 768 & 8 & (tested-positive; tested-negative) & $(34.84,66.16)$ & 1.90 \\
\hline Iris0 & 150 & 4 & (Iris-Setosa; remainder) & $(33.33,66.67)$ & 2.00 \\
\hline Glass0 & 214 & 9 & (build-win-float-proc; remainder) & $(32.71,67.29)$ & 2.06 \\
\hline Yeast1 & 1484 & 8 & (nuc; remainder) & $(28.91,71.09)$ & 2.46 \\
\hline Vehicle1 & 846 & 18 & (Saab; remainder) & $(28.37,71.63)$ & 2.52 \\
\hline Vehicle2 & 846 & 18 & (Bus; remainder) & $(28.37,71.63)$ & 2.52 \\
\hline Vehicle3 & 846 & 18 & (Opel; remainder) & $(28.37,71.63)$ & 2.52 \\
\hline Haberman & 306 & 3 & (Die; Survive) & $(27.42,73.58)$ & 2.68 \\
\hline Glass0123vs456 & 214 & 9 & (non-window glass; remainder) & $(23.83,76.17)$ & 3.19 \\
\hline Vehicle0 & 846 & 18 & (Van; remainder) & $(23.64,76.36)$ & 3.23 \\
\hline Ecoli1 & 336 & 7 & (im; remainder) & $(22.92,77.08)$ & 3.36 \\
\hline New-thyroid2 & 215 & 5 & (hypo; remainder) & $(16.89,83.11)$ & 4.92 \\
\hline New-thyroid1 & 215 & 5 & (hyper; remainder) & $(16.28,83.72)$ & 5.14 \\
\hline Ecoli2 & 336 & 7 & (pp; remainder) & $(15.48,84.52)$ & 5.46 \\
\hline Segment0 & 2308 & 19 & (brickface; remainder) & $(14.26,85.74)$ & 6.01 \\
\hline Glass6 & 214 & 9 & (headlamps; remainder) & $(13.55,86.45)$ & 6.38 \\
\hline Yeast3 & 1484 & 8 & (me3; remainder) & $(10.98,89.02)$ & 8.11 \\
\hline Ecoli3 & 336 & 7 & (imU; remainder) & $(10.88,89.12)$ & 8.19 \\
\hline Page-blocks0 & 5472 & 10 & (remainder; text) & $(10.23,89.77)$ & 8.77 \\
\hline Ecoli034vs5 & 200 & 7 & $(\mathrm{p}, \mathrm{imL}, \mathrm{imU} ; \mathrm{om})$ & $(10.00,90.00)$ & 9.00 \\
\hline Yeast2vs4v & 514 & 8 & (cyt; me2) & $(9.92,90.08)$ & 9.08 \\
\hline Ecoli067vs35 & 222 & 7 & $(\mathrm{cp}, \mathrm{omL}, \mathrm{pp} ; \mathrm{imL}, \mathrm{om})$ & $(9.91,90.09)$ & 9.09 \\
\hline Ecoli0234vs5 & 202 & 7 & (cp,ims,imL,imU; om) & $(9.90,90.10)$ & 9.10 \\
\hline Glass015vs2 & 172 & 9 & $\begin{array}{l}\text { (build-win-non float-proc,tableware, } \\
\text { build-win-float-proc; ve-win-float-proc) }\end{array}$ & $(9.88,90.12)$ & 9.12 \\
\hline Yeast0359vs78 & 506 & 8 & (mit,me1,me3,erl; vac,pox) & $(9.88,90.12)$ & 9.12 \\
\hline Yeast02579vs368 & 1004 & 8 & (mit,cyt,me3,vac,erl; me1,exc,pox) & $(9.86,90.14)$ & 9.14 \\
\hline Yeast0256vs3789 & 1004 & 8 & (mit,cyt,me3,exc; me1,vac,pox,erl) & $(9.86,90.14)$ & 9.14 \\
\hline Ecoli046vs5 & 203 & 6 & $(\mathrm{cp}, \mathrm{imU}, \mathrm{omL} ; \mathrm{om})$ & $(9.85,90.15)$ & 9.15 \\
\hline Ecoli01vs235 & 244 & 7 & $(\mathrm{cp}, \mathrm{im} ; \mathrm{imS}, \mathrm{imL}, \mathrm{om})$ & $(9.83,90.17)$ & 9.17 \\
\hline Ecoli0267vs35 & 224 & 7 & (cp,ims,omL,pp; imL,om) & $(9.82,90.18)$ & 9.18 \\
\hline Glass04vs5 & 92 & 9 & (build-win-float-proc,containers; tableware) & $(9.78,90.22)$ & 9.22 \\
\hline Ecoli0346vs5 & 205 & 7 & (cp,imL,imU,omL; om) & $(9.76,90.24)$ & 9.25 \\
\hline Ecoli0347vs56 & 257 & 7 & $(\mathrm{cp}, \mathrm{imL}, \mathrm{imU}, \mathrm{pp} ; \mathrm{om}, \mathrm{omL})$ & $(9.73,90.27)$ & 9.28 \\
\hline Yeast05679vs4 & 528 & 8 & (me2; mit,me3,exc,vac,erl) & $(9.66,90.34)$ & 9.35 \\
\hline Ecoli067vs5 & 220 & 6 & $(\mathrm{cp}, \mathrm{omL}, \mathrm{pp} ; \mathrm{om})$ & $(9.09,90.91)$ & 10.00 \\
\hline Vowel0 & 988 & 13 & (hid; remainder) & $(9.01,90.99)$ & 10.10 \\
\hline Glass016vs2 & 192 & 9 & $\begin{array}{l}\text { (ve-win-float-proc; build-win-float-proc, } \\
\text { build-win-non float-proc,headlamps) }\end{array}$ & $(8.89,91.11)$ & 10.29 \\
\hline Glass2 & 214 & 9 & (Ve-win-float-proc; remainder) & $(8.78,91.22)$ & 10.39 \\
\hline Ecoli0147vs2356 & 336 & 7 & $(\mathrm{cp}, \mathrm{im}, \mathrm{imU}, \mathrm{pp} ; \mathrm{imS}, \mathrm{imL}, \mathrm{om}, \mathrm{omL})$ & $(8.63,91.37)$ & 10.59 \\
\hline Led7digit02456789vs1 & 443 & 7 & $(0,2,4,5,6,7,8,9 ; 1)$ & $(8.35,91.65)$ & 10.97 \\
\hline
\end{tabular}

\subsection{FURIA for imbalanced data}

This section is devoted to develop a detailed performance study on FURIA_CS. Three different aspects of the proposed extension of FURIA to imbalanced problems are analyzed: 
Table 3 (Continued).

\begin{tabular}{|c|c|c|c|c|c|}
\hline Data-sets & \#Ex. & \#Atts. & Class $(-,+)$ & \%Class $(-,+)$ & IR \\
\hline Glass06vs5 & 108 & 9 & (build-win-float-proc,headlamps; tableware) & $(8.33,91.67)$ & 11.00 \\
\hline Ecoli01vs5 & 240 & 6 & $(\mathrm{cp}, \mathrm{im} ; \mathrm{om})$ & $(8.33,91.67)$ & 11.00 \\
\hline Glass0146vs2 & 205 & 9 & $\begin{array}{l}\text { (build-win-float-proc,containers, headlamps, } \\
\text { build-win-non float-proc; } v e-w i n-f l o a t-p r o c)\end{array}$ & $(8.29,91.71)$ & 11.06 \\
\hline Ecoli0147vs56 & 332 & 6 & $(\mathrm{cp}, \mathrm{im}, \mathrm{imU}, \mathrm{pp} ; \mathrm{om}, \mathrm{omL})$ & $(7.53,92.47)$ & 12.28 \\
\hline Cleveland0vs4 & 177 & 13 & $(0 ; 4)$ & $(7.34,92.66)$ & 12.62 \\
\hline Ecoli0146vs5 & 280 & 6 & $(\mathrm{cp}, \mathrm{im}, \mathrm{imU}, \mathrm{omL} ; \mathrm{om})$ & $(7.14,92.86)$ & 13.00 \\
\hline Ecoli4 & 336 & 7 & (om; remainder) & $(6.74,93.26)$ & 13.84 \\
\hline Yeast1vs7 & 459 & 8 & (nuc; vac) & $(6.72,93.28)$ & 13.87 \\
\hline Shuttle0vs4 & 1829 & 9 & (Rad Flow; Bypass) & $(6.72,93.28)$ & 13.87 \\
\hline Glass4 & 214 & 9 & (containers; remainder) & $(6.07,93.93)$ & 15.47 \\
\hline Page-blocks13vs2 & 472 & 10 & (graphic; horiz.line,picture) & $(5.93,94.07)$ & 15.85 \\
\hline Glass016vs5 & 184 & 9 & $\begin{array}{c}\text { (tableware; build-win-float-proc, } \\
\text { build-win-non float-proc,headlamps) }\end{array}$ & $(4.89,95.11)$ & 19.44 \\
\hline Shuttle2vs 4 & 129 & 9 & (Fpv Open; Bypass) & $(4.65,95.35)$ & 20.5 \\
\hline Yeast1458vs7 & 693 & 8 & (vac; nuc,me $2, \operatorname{me} 3, \mathrm{pox}$ ) & $(4.33,95.67)$ & 22.10 \\
\hline Glass5 & 214 & 9 & (tableware; remainder) & $(4.20,95.80)$ & 22.81 \\
\hline Yeast2vs8 & 482 & 8 & (pox; cyt) & $(4.15,95.85)$ & 23.10 \\
\hline Yeast4 & 1484 & 8 & (me $2 ;$ remainder) & $(3.43,96.57)$ & 28.41 \\
\hline Yeast1289vs7 & 947 & 8 & (vac; nuc,cyt,pox,erl) & $(3.17,96.83)$ & 30.56 \\
\hline Yeast5 & 1484 & 8 & (me1; remainder) & $(2.96,97.04)$ & 32.78 \\
\hline Ecoli0137vs26 & 281 & 7 & $(\mathrm{pp}, \mathrm{imL} ; \mathrm{cp}, \mathrm{im}, \mathrm{imU}, \mathrm{imS})$ & $(2.49,97.51)$ & 39.15 \\
\hline Yeast6 & 1484 & 8 & (exc; remainder) & $(2.49,97.51)$ & 39.15 \\
\hline
\end{tabular}

Table 4. Cost matrix for a two-class problem.

\begin{tabular}{c|c|c}
\hline & Positive Class & Negative Class \\
\hline Positive Prediction & 0 & $1 / \mathrm{IR}$ \\
Negative Prediction & 1 & $\mathrm{PF}$ \\
\hline
\end{tabular}

(1) The performance of FURIA_CS with respect to different penalization factors in the misclassification costs matrix.

(2) The performance of FURIA_CS (an internal method) against the combination of FURIA and preprocessing (the counterpart external methods): FURIA+SMOTE and FURIA+SMOTE+ENN.

(3) The influence of some design decisions in the performance of FURIA_CS.

\subsubsection{Penalization factors in the cost matrix}

As mentioned, there are cases where it makes sense to add a penalty to correct classifications of instances to the negative class. ${ }^{45}$ In this study, two different heuristic values will be considered standing for a low and high penalization:

$$
\begin{aligned}
& \mathrm{PF}_{1}=\frac{1}{2 \mathrm{IR}} \\
& \mathrm{PF}_{2}=1-\frac{1}{\mathrm{IR}}
\end{aligned}
$$


Table 5. Choice of parameters for the algorithms considered in the experimentation.

\begin{tabular}{|c|c|}
\hline Algorithm Family & Parameters \\
\hline $\mathrm{C} 4.5$ & $\begin{array}{l}- \text { pruned }=\text { True } \\
- \text { confidence }=0.25 \\
- \text { minimum number of item-sets per leaf }=2 \\
-C(+,-)=\mathrm{IR}, C(-,+)=1, C(+,+)=0, C(-,-)=0\end{array}$ \\
\hline SVM & $\begin{array}{l}\text { - kernel type }=\text { polynomial } \\
-C=100 \\
- \text { tolerance of termination criterion }=0.001 \\
- \text { degree (for kernel function) }=1 \\
- \text { gamma (for kernel function) }=0.01 \\
-\operatorname{coef}() \text { (for kernel funcion) }=0 \\
- \text { use shrinking heuristics }=\text { true } \\
-C(+,-)=\mathrm{IR}, C(-,+)=1, C(+,+)=0, C(-,-)=0\end{array}$ \\
\hline $\mathrm{k}-\mathrm{NN}$ & $\begin{array}{l}-\mathrm{k}=3 \\
- \text { distance }=\text { Heterogeneous Value Difference Metric (HVDM) } \\
-C(+,-)=\mathrm{IR}, C(-,+)=1, C(+,+)=0, C(-,-)=0\end{array}$ \\
\hline FH-GBML & $\begin{aligned}- & \text { conjunction operator }=\text { product t-norm } \\
- & \text { rule weight }=\text { PCF }(\mathrm{FH}-\mathrm{GBML} \text { and } \mathrm{FH}-\mathrm{GBML}+\text { preprocessing }) \\
& \text { and PCF-SC }{ }^{2}(\mathrm{FH}-\mathrm{GBML}-\mathrm{CS}) \\
- & \text { fuzzy reasoning method }=\text { winning rule } \\
- & \text { number of fuzzy rules }=5 . \mathrm{d} \text { (max. } 50 \text { rules }) \\
- & \text { number of rule sets }=200 \\
- & \text { crossover probability }=0.9 \\
- & \text { mutation probability }=1 / \mathrm{d} \\
- & \text { number of replaced rules }=\text { all rules except the best-one } \\
& (\text { Pittsburgh-part, elitist approach) and number of rules } / 5 \text { (GCCL-part) } \\
- & \text { total number of generations }=1000 \\
- & \text { don't care probability }=0.5 \\
- & \text { probability of the application of the GCCL iteration }=0.5 \\
- & C(+,-)=\mathrm{IR}, C(-,+)=1, C(+,+)=0, C(-,-)=0\end{aligned}$ \\
\hline
\end{tabular}

Table 6. AUC-based comparison of FURIA and FURIA_CS with penalization factors $\mathrm{PF}_{0}=0, \mathrm{PF}_{1}=1 /(2 \mathrm{IR}), \mathrm{PF}_{2}=1-(1 / \mathrm{IR})$.

\begin{tabular}{ccccc}
\hline Datasets & $\mathrm{AUC}-\mathrm{FURIA}$ & $\mathrm{AUC}-\mathrm{PF}_{0}$ & $\mathrm{AUC}-\mathrm{PF}_{1}$ & $\mathrm{AUC}-\mathrm{PF}_{2}$ \\
\hline $1.82 \leq \mathrm{IR}<9(22)$ & 0.837 & 0.866 & 0.875 & $\mathbf{0 . 8 8 0}$ \\
$9 \leq \mathrm{IR}<11(21)$ & 0.776 & 0.802 & 0.823 & $\mathbf{0 . 8 3 2}$ \\
$11 \leq \mathrm{IR} \leq 39.15(21)$ & 0.771 & 0.795 & $\mathbf{0 . 8 4 5}$ & 0.825 \\
\hline Average & 0.794 & 0.821 & $\mathbf{0 . 8 4 7}$ & 0.846 \\
\hline
\end{tabular}

The summarized of the average results of FURIA, FURIA-CS with penalizations $0, \mathrm{PF}_{1}$ and $\mathrm{PF}_{2}$, relative to the AUC metric (Eq. (3)), are shown in Table 6. The same study is shown in Table 7 for the GM metric. In view of the obtained results, non null penalty factors are preferred. The $p$-value of the Friedman Rank Sum Test 
Table 7. GM-based comparison of FURIA and FURIA_CS with penalization factors $\mathrm{PF}_{0}=0, \mathrm{PF}_{1}=1 /(2 \mathrm{IR}), \mathrm{PF}_{2}=1-(1 / \mathrm{IR})$.

\begin{tabular}{ccccc}
\hline Datasets & GM - FURIA & GM $-\mathrm{PF}_{0}$ & $\mathrm{GM}-\mathrm{PF}_{1}$ & $\mathrm{GM}-\mathrm{PF}_{2}$ \\
\hline $1.82 \leq \mathrm{IR}<9(22)$ & 0.850 & 0.857 & 0.865 & $\mathbf{0 . 8 7 6}$ \\
$9 \leq \mathrm{IR}<11(21)$ & 0.711 & 0.744 & 0.773 & $\mathbf{0 . 8 0 0}$ \\
$11 \leq \mathrm{IR} \leq 39.15(21)$ & 0.662 & 0.696 & $\mathbf{0 . 7 9 0}$ & 0.782 \\
\hline Average & 0.741 & 0.765 & 0.809 & $\mathbf{0 . 8 1 9}$ \\
\hline
\end{tabular}

is 0.042 , showing the relevance of the choice of $\mathrm{PF}$ (95\% confidence level). $\mathrm{FP}_{2}$ is preferred in low or medium imbalanced datasets $(1.82 \leq \mathrm{IR}<11)$. $\mathrm{FP}_{1}$ performs better for highly imbalanced problems $(11 \leq \mathrm{IR} \leq 39.15)$. See Tables 19 and 20 in the Appendix for detailed results.

\subsubsection{FURIA_CS vs. the combination of FURIA and preprocessing}

In Table 8, AUC-based performances of the original FURIA algorithm, FURIA combined with two preprocessing methods (SMOTE and SMOTE+ENN), and FURIA_CS are displayed. FURIA_CS outperforms the other approaches (the statistical relevance of the differences will be analyzed later). Notice this result seems to contradict the conclusions of recent references, ${ }^{32}$ however in these works a different cost-sensitive algorithm was used and the diagonal of the cost matrix was assumed to be zero. Differences between SMOTE and SMOTE+ENN were not significant.

In Table 9 the ranking of the four algorithms on each dataset is shown. FURIA_CS appears 31 times in the first position, 22 times in the second position, 10 times in the third, and only 1 time in the last position. Those 11 datasets where FURIA_CS was in the third or fourth positions are marked in boldface. Table 10 displays the mean rankings of each algorithm, as part of the Friedman tests used for assessing the statistical significance of the differences. ${ }^{12}$

The mean value of the AUC in all problems is shown in Table 8. The dispersion of the results is illustrated with the help of box plots (see Fig. 1). The differences are relevant according to the Friedman test. The $p$-values of the paired comparisons between the best ranked algorithm and the alternatives are in Table 11. A Wilcoxon test has been used to assess these differences.

The conclusions of this part of the study are:

- Equal means hypothesis is rejected in favour of FURIA_CS, FURIA + SMOTE, and FURIA + SMOTE + ENN with respect to FURIA, as expected.

- Equal means hypothesis is rejected in favour of FURIA_CS with respect to FURIA + SMOTE and FURIA + SMOTE + ENN.

- Equal means hypothesis is not rejected in the case of FURIA + SMOTE vs. FURIA + SMOTE + ENN. 
Table 8. Comparison between FURIA_CS and FURIA with and without preprocessing methods in terms of sst $_{\mathrm{AUC}}$.

\begin{tabular}{|c|c|c|c|c|}
\hline Dataset & $\begin{array}{l}\text { FURIA } \\
\text { Tst }_{\text {AUC }}\end{array}$ & $\begin{array}{c}\text { FURIA + SMOTE } \\
\text { Tst }{ }_{\text {AUC }}\end{array}$ & $\begin{array}{c}\text { FURIA }+ \text { SMOTE + ENN } \\
\text { Tst }_{\text {AUC }}\end{array}$ & $\begin{array}{c}\text { FURIA_CS } \\
\text { TstaUC }\end{array}$ \\
\hline Glass1 & 0.704 & 0.773 & 0.762 & 0.780 \\
\hline EcoliOvs1 & 0.986 & 0.979 & 0.979 & 0.986 \\
\hline Wisconsin & 0.960 & 0.963 & 0.965 & 0.978 \\
\hline Pima & 0.672 & 0.729 & 0.745 & 0.736 \\
\hline Iris0 & 1.000 & 1.000 & 1.000 & 1.000 \\
\hline Glass0 & 0.797 & 0.806 & 0.845 & 0.836 \\
\hline Yeast1 & 0.668 & 0.719 & 0.706 & 0.707 \\
\hline Vehicle1 & 0.650 & 0.720 & 0.766 & 0.770 \\
\hline Vehicle2 & 0.969 & 0.975 & 0.959 & 0.977 \\
\hline Vehicle3 & 0.653 & 0.749 & 0.794 & 0.785 \\
\hline Haberman & 0.577 & 0.639 & 0.624 & 0.687 \\
\hline Glass0123vs456 & 0.868 & 0.909 & 0.913 & 0.903 \\
\hline Vehicle0 & 0.929 & 0.952 & 0.940 & 0.946 \\
\hline Ecoli1 & 0.840 & 0.901 & 0.872 & 0.886 \\
\hline New-thyroid2 & 0.937 & 0.965 & 0.960 & 0.951 \\
\hline New-thyroid 1 & 0.948 & 0.977 & 0.986 & 0.963 \\
\hline Ecoli2 & 0.856 & 0.899 & 0.866 & 0.917 \\
\hline Segment0 & 0.987 & 0.500 & 0.500 & 0.992 \\
\hline Glass6 & 0.841 & 0.886 & 0.919 & 0.908 \\
\hline Yeast3 & 0.877 & 0.922 & 0.913 & 0.921 \\
\hline Ecoli3 & 0.769 & 0.831 & 0.859 & 0.855 \\
\hline Page-blocks0 & 0.929 & 0.952 & 0.947 & 0.942 \\
\hline Ecoli034vs5 & 0.819 & 0.901 & 0.867 & 0.891 \\
\hline Yeast2vs $4 \mathrm{v}$ & 0.825 & 0.873 & 0.847 & 0.895 \\
\hline Ecoli067vs35 & 0.877 & 0.880 & 0.818 & 0.872 \\
\hline Ecoli0234vs5 & 0.838 & 0.848 & 0.895 & 0.880 \\
\hline Glass015vs2 & 0.526 & 0.750 & 0.763 & 0.615 \\
\hline Yeast0359vs78 & 0.584 & 0.697 & 0.672 & 0.715 \\
\hline Yeast02579vs368 & 0.895 & 0.898 & 0.887 & 0.915 \\
\hline Yeast0256vs3789 & 0.688 & 0.753 & 0.789 & 0.792 \\
\hline Ecoli046vs5 & 0.816 & 0.885 & 0.849 & 0.889 \\
\hline Ecoli01vs235 & 0.735 & 0.821 & 0.816 & 0.825 \\
\hline Ecoli0267vs35 & 0.802 & 0.847 & 0.823 & 0.827 \\
\hline Glass04vs5 & 0.994 & 0.979 & 0.979 & 0.994 \\
\hline Ecoli0346vs5 & 0.841 & 0.932 & 0.902 & 0.897 \\
\hline Ecoli0347vs56 & 0.815 & 0.899 & 0.901 & 0.769 \\
\hline Yeast05679vs4 & 0.696 & 0.814 & 0.780 & 0.801 \\
\hline Ecoli067vs5 & 0.840 & 0.847 & 0.849 & 0.865 \\
\hline Vowel0 & 0.950 & 0.958 & 0.956 & 0.966 \\
\hline Glass016vs2 & 0.519 & 0.631 & 0.736 & 0.635 \\
\hline Glass2 & 0.558 & 0.662 & 0.702 & 0.738 \\
\hline Ecoli0147vs2356 & 0.821 & 0.866 & 0.896 & 0.845 \\
\hline Led7digit02456789vs1 & 0.881 & 0.884 & 0.858 & 0.908 \\
\hline Glass06vs5 & 0.945 & 0.971 & 0.977 & 0.945 \\
\hline Ecoli01vs5 & 0.838 & 0.798 & 0.869 & 0.861 \\
\hline Glass0146vs2 & 0.497 & 0.760 & 0.688 & 0.740 \\
\hline Ecoli0147vs56 & 0.796 & 0.879 & 0.852 & 0.865 \\
\hline Cleveland0vs4 & 0.748 & 0.500 & 0.500 & 0.751 \\
\hline Ecoli0146vs5 & 0.744 & 0.874 & 0.845 & 0.865 \\
\hline Ecoli4 & 0.815 & 0.860 & 0.829 & 0.864 \\
\hline Yeast1vs7 & 0.546 & 0.671 & 0.701 & 0.690 \\
\hline Shuttle0vs 4 & 1.000 & 1.000 & 1.000 & 1.000 \\
\hline Glass4 & 0.870 & 0.889 & 0.881 & 0.935 \\
\hline Page-blocks13vs2 & 0.997 & 0.992 & 0.992 & 0.997 \\
\hline Glass016vs5 & 0.844 & 0.921 & 0.812 & 0.888 \\
\hline Shuttle2vs4 & 0.950 & 0.995 & 1.000 & 0.950 \\
\hline Yeast1458vs7 & 0.493 & 0.521 & 0.531 & 0.649 \\
\hline Glass5 & 0.847 & 0.942 & 0.828 & 0.897 \\
\hline Yeast2vs 8 & 0.773 & 0.718 & 0.783 & 0.773 \\
\hline Yeast4 & 0.545 & 0.720 & 0.770 & 0.869 \\
\hline Yeast1289vs7 & 0.566 & 0.599 & 0.535 & 0.771 \\
\hline Yeast5 & 0.885 & 0.914 & 0.965 & 0.965 \\
\hline Ecoli0137vs26 & 0.746 & 0.833 & 0.831 & 0.848 \\
\hline Yeast6 & 0.739 & 0.805 & 0.813 & 0.892 \\
\hline Total Mean & 0.796 & 0.836 & 0.834 & 0.858 \\
\hline
\end{tabular}


Table 9. Performance ranking of FURIA_CS and FURIA with and without preprocessing methods in terms of Tst AUC.

\begin{tabular}{|c|c|c|c|c|}
\hline Dataset & $1 \mathrm{st}$ & $2 \mathrm{nd}$ & $3 \mathrm{rd}$ & 4 th \\
\hline Glass1 & $\mathrm{CS}$ & SMOTE & SMOTE+ENN & ORIGINAL \\
\hline EcoliOvs1 & CS/ORIGINAL & SMOTE/SMOTE+ENN & - & - \\
\hline Wisconsin & CS & SMOTE+ENN & SMOTE & ORIGINAL \\
\hline Pima & SMOTE+ENN & $\mathrm{CS}$ & SMOTE & ORIGINAL \\
\hline Iris0 & ALL & - & - & - \\
\hline Glass0 & SMOTE+ENN & CS & SMOTE & ORIGINAL \\
\hline Yeast1 & SMOTE & CS & SMOTE+ENN & ORIGINAL \\
\hline Vehicle1 & CS & SMOTE+ENN & SMOTE & ORIGINAL \\
\hline Vehicle2 & CS & SMOTE & ORIGINAL & $\mathrm{SMOTE}+\mathrm{ENN}$ \\
\hline Vehicle3 & SMOTE+ENN & $\mathrm{CS}$ & SMOTE & ORIGINAL \\
\hline Haberman & CS & SMOTE & SMOTE+ENN & ORIGINAL \\
\hline Glass0123vs 456 & SMOTE+ENN & SMOTE & CS & ORIGINAL \\
\hline Vehicle0 & SMOTE & CS & SMOTE+ENN & ORIGINAL \\
\hline Ecoli1 & SMOTE & CS & SMOTE+ENN & ORIGINAL \\
\hline New-thyroid2 & SMOTE & SMOTE+ENN & CS & ORIGINAL \\
\hline New-thyroid1 & SMOTE+ENN & SMOTE & CS & ORIGINAL \\
\hline Ecoli2 & CS & SMOTE & SMOTE+ENN & ORIGINAL \\
\hline Segment0 & CS & ORIGINAL & SMOTE/SMOTE+ENN & - \\
\hline Glass6 & SMOTE+ENN & $\mathrm{CS}$ & SMOTE & ORIGINAL \\
\hline Yeast3 & SMOTE & CS & SMOTE+ENN & ORIGINAL \\
\hline Ecoli3 & SMOTE+ENN & CS & SMOTE & ORIGINAL \\
\hline Page-blocks0 & SMOTE & SMOTE+ENN & $\mathrm{CS}$ & ORIGINAL \\
\hline Ecoli034vs5 & SMOTE & $\mathrm{CS}$ & SMOTE+ENN & ORIGINAL \\
\hline Yeast2vs4v & CS & SMOTE & SMOTE+ENN & ORIGINAL \\
\hline Ecoli067vs35 & SMOTE & ORIGINAL & $\mathrm{CS}$ & SMOTE+ENN \\
\hline Ecoli0234vs5 & $\mathrm{SMOTE}+\mathrm{ENN}$ & $\mathrm{CS}$ & SMOTE & ORIGINAL \\
\hline Glass015vs2 & SMOTE+ENN & SMOTE & CS & ORIGINAL \\
\hline Yeast0359vs78 & $\mathrm{CS}$ & SMOTE & SMOTE+ENN & ORIGINAL \\
\hline Yeast02579vs368 & CS & SMOTE & SMOTE+ENN & ORIGINAL \\
\hline Yeast0256vs3789 & CS & SMOTE+ENN & SMOTE & ORIGINAL \\
\hline Ecoli046vs5 & CS & SMOTE & SMOTE+ENN & ORIGINAL \\
\hline Ecoli01vs 235 & CS & SMOTE & SMOTE+ENN & ORIGINAL \\
\hline Ecoli0267vs35 & SMOTE & CS & SMOTE+ENN & ORIGINAL \\
\hline Glass04vs5 & CS/ORIGINAL & SMOTE/SMOTE+ENN & - & - \\
\hline Ecolio346vs5 & SMOTE & SMOTE+ENN & CS & ORIGINAL \\
\hline Ecoli0347vs56 & SMOTE+ENN & SMOTE & ORIGINAL & CS \\
\hline Yeast05679vs 4 & SMOTE & $\mathrm{CS}$ & SMOTE+ENN & ORIGINAL \\
\hline Ecoli067vs5 & CS & SMOTE+ENN & SMOTE & ORIGINAL \\
\hline Vowel0 & $\mathrm{CS}$ & SMOTE & SMOTE+ENN & ORIGINAL \\
\hline Glass016vs2 & SMOTE+ENN & CS & SMOTE & ORIGINAL \\
\hline Glass2 & CS & $\mathrm{SMOTE}+\mathrm{ENN}$ & SMOTE & ORIGINAL \\
\hline Ecoli0147vs2356 & SMOTE+ENN & SMOTE & CS & ORIGINAL \\
\hline Led7digit02456789vs1 & CS & SMOTE & ORIGINAL & SMOTE+ENN \\
\hline Glass06vs5 & SMOTE+ENN & SMOTE & CS/ORIGINAL & - \\
\hline Ecoli01vs5 & SMOTE+ENN & $\mathrm{CS}$ & ORIGINAL & SMOTE \\
\hline Glass0146vs2 & SMOTE & CS & SMOTE+ENN & ORIGINAL \\
\hline Ecoli0147vs56 & SMOTE & CS & SMOTE+ENN & ORIGINAL \\
\hline Cleveland0vs4 & CS & ORIGINAL & SMOTE+ENN/SMOTE & - \\
\hline Ecoli0146vs5 & SMOTE & CS & SMOTE+ENN & ORIGINAL \\
\hline Ecoli4 & $\mathrm{CS}$ & SMOTE & SMOTE+ENN & ORIGINAL \\
\hline Yeast1vs7 & SMOTE+ENN & CS & SMOTE & ORIGINAL \\
\hline Shuttle0vs 4 & ALL & - & - & - \\
\hline Glass4 & $\mathrm{CS}$ & SMOTE & SMOTE+ENN & ORIGINAL \\
\hline Page-blocks13vs2 & CS & ORIGINAL & SMOTE+ENN/SMOTE & - \\
\hline Glass016vs5 & SMOTE & CS & ORIGINAL & SMOTE+ENN \\
\hline Shuttle2vs4 & SMOTE+ENN & SMOTE & CS/ORIGINAL & - \\
\hline Yeast1458vs7 & CS & SMOTE+ENN & SMOTE & ORIGINAL \\
\hline Glass5 & SMOTE & $\mathrm{CS}$ & ORIGINAL & $\mathrm{SMOTE}+\mathrm{ENN}$ \\
\hline Yeast2vs8 & SMOTE+ENN & CS & ORIGINAL & SMOTE \\
\hline Yeast4 & CS & SMOTE+ENN & SMOTE & ORIGINAL \\
\hline Yeast1289vs7 & CS & SMOTE & ORIGINAL & SMOTE+ENN \\
\hline Yeast5 & CS/SMOTE+ENN & SMOTE & ORIGINAL & - \\
\hline Ecoli0137vs26 & $\mathrm{CS}$ & SMOTE & SMOTE+ENN & ORIGINAL \\
\hline Yeast6 & CS & SMOTE+ENN & SMOTE & ORIGINAL \\
\hline
\end{tabular}


Table 10. Ranking obtained through Friedman's test.

\begin{tabular}{cc}
\hline Algorithm & Ranking \\
\hline CS & 1.703 \\
SMOTE & 2.078 \\
SMOTE_ENN & 2.265 \\
ORIGINAL & 3.515 \\
\hline
\end{tabular}

Table 11. Wilcoxon's test for comparing FURIA with and without preprocessing approaches and cost-sensitive learning.

\begin{tabular}{lcc}
\hline \multicolumn{1}{c}{ Comparison } & $p$-value & Hypothesis $(\alpha=0.05)$ \\
\hline FURIA_CS vs. FURIA & $3.1 \mathrm{e}-10$ & Reject \\
FURIA_SMOTE vs. FURIA & $5.6 \mathrm{e}-08$ & Reject \\
FURIA_SMOTE + ENN vs. FURIA & $7.3 \mathrm{e}-07$ & Reject \\
\hline FURIA_CS vs. FURIA + SMOTE & 0.017 & Reject \\
FURIA_CS vs. FURIA + SMOTE+ENN & 0.0027 & Reject \\
\hline FURIA+SMOTE + ENN vs. FURIA + SMOTE & 0.72 & Not reject \\
\hline
\end{tabular}

\subsubsection{Influence of some design decisions in the performance of FURIA_CS}

According to Ref. 15, the splitting criteria in decision trees is not sensitive to costs. If the conclusions of that reference could be applied to fuzzy classification rule learning, the redefinition of Information Gain in Eq. (15) should not be needed, in contradiction with the postulates of the current study.

The experiments in Table 12 were designed for assessing the need of a cost-adapted information gain criterion in FURIA_CS. The results in column "FURIA_CS-IG" were computed after reverting Eq. (15) to its original definition (Eq. (9)). Since FURIA_CS-IG is significantly inferior to FURIA_CS and not different than FURIA combined with SMOTE or SMOTE + ENN, Table 12 actually shows that the cost-based definition proposed in Eq. (15) significantly contributes to the performance of the new cost-sensitive algorithm.

In another work related to decision tree learning ${ }^{6}$ it was concluded that the pruning criteria are not relevant when designing a cost-sensitive algorithm. As done before, the algorithm FURIA_CS_SP is built by removing the pruning stage from FURIA_CS (Eqs. (21) and (22)). In Table 13 the results of FURIA_CS and FURIA_CS_SP are compared, showing a small advantage in favor of FURIA_CS. Nevertheless, the difference found is actually less relevant than that found for the Information Gain study.

\subsection{Comparison between FURIA_CS and other classification algorithms}

The compared results of FURIA_CS, C4.5, ${ }^{41} \mathrm{SVM}^{46}{ }^{46} \mathrm{k}-\mathrm{NN},{ }^{8}$ and FH-GBML rule generation algorithm ${ }^{28}$ are shown in Table 14 . The cost-sensitive version of each technique was used, along with SMOTE and SMOTE + ENN preprocessed datasets combined with error-based versions of the algorithms. ${ }^{32}$ 
Table 12. Comparison between FURIA + preprocessing approaches and FURIA_CS with $\mathrm{IG}=9$.

\begin{tabular}{|c|c|c|c|}
\hline Dataset & $\begin{array}{c}\text { FURIA_CS-IG } \\
\text { TstAUC }\end{array}$ & $\begin{array}{c}\text { FURIA + SMOTE } \\
\text { Tst }{ }_{\text {AUC }}\end{array}$ & $\begin{array}{c}\text { FURIA }+ \text { SMOTE }+ \text { ENN } \\
\text { Tst }_{\text {AUC }}\end{array}$ \\
\hline Glass1 & 0.804 & 0.773 & 0.762 \\
\hline EcoliOvs1 & 0.986 & 0.979 & 0.979 \\
\hline Wisconsin & 0.974 & 0.963 & 0.965 \\
\hline Pima & 0.809 & 0.729 & 0.745 \\
\hline Iris0 & 1.000 & 1.000 & 1.000 \\
\hline Glass0 & 0.825 & 0.806 & 0.845 \\
\hline Yeast 1 & 0.736 & 0.719 & 0.706 \\
\hline Vehicle1 & 0.788 & 0.720 & 0.766 \\
\hline Vehicle2 & 0.964 & 0.975 & 0.959 \\
\hline Vehicle3 & 0.800 & 0.749 & 0.794 \\
\hline Haberman & 0.759 & 0.639 & 0.624 \\
\hline Glass $0123 v s 456$ & 0.907 & 0.909 & 0.913 \\
\hline Vehicle0 & 0.927 & 0.952 & 0.940 \\
\hline Ecoli1 & 0.880 & 0.901 & 0.872 \\
\hline New-thyroid2 & 0.951 & 0.965 & 0.960 \\
\hline New-thyroid1 & 0.982 & 0.977 & 0.986 \\
\hline Ecoli2 & 0.865 & 0.899 & 0.866 \\
\hline Segmento & 0.985 & 0.5 & 0.5 \\
\hline Glass6 & 0.907 & 0.886 & 0.919 \\
\hline Yeast3 & 0.904 & 0.922 & 0.913 \\
\hline Ecoli3 & 0.769 & 0.831 & 0.859 \\
\hline Page-blocks0 & 0.932 & 0.952 & 0.947 \\
\hline Ecoli034vs5 & 0.888 & 0.901 & 0.867 \\
\hline Yeast2vs $4 \mathrm{v}$ & 0.918 & 0.873 & 0.847 \\
\hline Ecoli067vs35 & 0.852 & 0.880 & 0.818 \\
\hline Ecoli0234vs5 & 0.914 & 0.848 & 0.895 \\
\hline Glass015vs2 & 0.625 & 0.750 & 0.763 \\
\hline Yeast0359vs78 & 0.640 & 0.697 & 0.672 \\
\hline Yeast $02579 v s 368$ & 0.914 & 0.898 & 0.887 \\
\hline Yeast0256vs3789 & 0.778 & 0.753 & 0.789 \\
\hline Ecoli046vs5 & 0.866 & 0.885 & 0.849 \\
\hline Ecoli01vs235 & 0.858 & 0.821 & 0.816 \\
\hline Ecoli0267vs35 & 0.822 & 0.847 & 0.823 \\
\hline Glass04vs5 & 0.994 & 0.979 & 0.979 \\
\hline Ecoli0346vs5 & 0.897 & 0.932 & 0.902 \\
\hline Ecoli0347vs56 & 0.811 & 0.899 & 0.901 \\
\hline Yeast05679vs4 & 0.722 & 0.814 & 0.780 \\
\hline Ecoli067vs5 & 0.842 & 0.847 & 0.849 \\
\hline Vowel0 & 0.953 & 0.958 & 0.956 \\
\hline Glass016vs2 & 0.583 & 0.631 & 0.736 \\
\hline Glass2 & 0.650 & 0.662 & 0.702 \\
\hline Ecoli0147vs2356 & 0.863 & 0.866 & 0.896 \\
\hline Led7digit02456789vs1 & 0.908 & 0.884 & 0.858 \\
\hline Glass06vs5 & 0.945 & 0.971 & 0.977 \\
\hline Ecoli01vs5 & 0.888 & 0.798 & 0.869 \\
\hline Glass0146vs2 & 0.641 & 0.760 & 0.688 \\
\hline Ecoli0147vs56 & 0.856 & 0.879 & 0.852 \\
\hline Cleveland0vs4 & 0.500 & 0.500 & 0.50 \\
\hline Ecoli0146vs5 & 0.865 & 0.874 & 0.845 \\
\hline Ecoli4 & 0.846 & 0.860 & 0.829 \\
\hline Yeast1vs7 & 0.579 & 0.671 & 0.701 \\
\hline Shuttle0vs4 & 1.000 & 1.000 & 1.000 \\
\hline Glass4 & 0.875 & 0.889 & 0.881 \\
\hline Page-blocks13vs2 & 0.978 & 0.992 & 0.992 \\
\hline Glass016vs5 & 0.844 & 0.921 & 0.812 \\
\hline Shuttle2vs4 & 0.950 & 0.995 & 1.000 \\
\hline Yeast1458vs7 & 0.509 & 0.521 & 0.531 \\
\hline Glass5 & 0.897 & 0.942 & 0.828 \\
\hline Yeast2vs8 & 0.773 & 0.718 & 0.783 \\
\hline Yeast4 & 0.655 & 0.720 & 0.770 \\
\hline Yeast1289vs7 & 0.566 & 0.599 & 0.535 \\
\hline Yeast5 & 0.919 & 0.914 & 0.965 \\
\hline Ecoli0137vs26 & 0.748 & 0.833 & 0.831 \\
\hline Yeast6 & 0.710 & 0.805 & 0.813 \\
\hline Total Mean & 0.837 & 0.836 & 0.834 \\
\hline
\end{tabular}


Table 13. Comparation between FURIA_CS and FURIA_CS-SP, without pruning stage.

\begin{tabular}{|c|c|c|}
\hline Dataset & $\begin{array}{c}\text { FURIA-CS } \\
\text { TstAUC }\end{array}$ & $\begin{array}{c}\text { FURIA-CS-SP } \\
\text { TstAUCM }\end{array}$ \\
\hline Glass1 & 0.780 & 0.757 \\
\hline Ecoli0vs1 & 0.986 & 0.986 \\
\hline Wisconsin & 0.978 & 0.978 \\
\hline Pima & 0.736 & 0.733 \\
\hline Iris0 & 1.000 & 1.000 \\
\hline Glass0 & 0.836 & 0.808 \\
\hline Yeast1 & 0.707 & 0.766 \\
\hline Vehicle1 & 0.770 & 0.763 \\
\hline Vehicle2 & 0.977 & 0.977 \\
\hline Vehicle3 & 0.785 & 0.781 \\
\hline Haberman & 0.687 & 0.687 \\
\hline Glass0123vs456 & 0.903 & 0.903 \\
\hline Vehicle0 & 0.946 & 0.944 \\
\hline Ecoli1 & 0.886 & 0.883 \\
\hline New-thyroid2 & 0.951 & 0.951 \\
\hline New-thyroid1 & 0.963 & 0.963 \\
\hline Ecoli2 & 0.917 & 0.917 \\
\hline Segment0 & 0.992 & 0.992 \\
\hline Glass6 & 0.908 & 0.908 \\
\hline Yeast3 & 0.921 & 0.917 \\
\hline Ecoli3 & 0.855 & 0.851 \\
\hline Page-blocks0 & 0.942 & 0.934 \\
\hline Ecoli034vs5 & 0.891 & 0.891 \\
\hline Yeast2vs4v & 0.895 & 0.885 \\
\hline Ecoli067vs35 & 0.872 & 0.875 \\
\hline Ecoli0234vs5 & 0.880 & 0.880 \\
\hline Glass015vs2 & 0.615 & 0.605 \\
\hline Yeast0359vs78 & 0.715 & 0.715 \\
\hline Yeast02579vs368 & 0.915 & 0.915 \\
\hline Yeast0256vs3789 & 0.792 & 0.792 \\
\hline Ecoli046vs5 & 0.889 & 0.864 \\
\hline Ecoli01vs235 & 0.825 & 0.807 \\
\hline Ecoli0267vs35 & 0.827 & 0.825 \\
\hline Glass04vs5 & 0.994 & 0.994 \\
\hline Ecoli0346vs5 & 0.897 & 0.897 \\
\hline Ecoli0347vs56 & 0.769 & 0.749 \\
\hline Yeast05679vs4 & 0.801 & 0.813 \\
\hline Ecoli067vs5 & 0.865 & 0.865 \\
\hline Vowel0 & 0.966 & 0.966 \\
\hline Glass016vs2 & 0.635 & 0.593 \\
\hline Glass2 & 0.738 & 0.738 \\
\hline Ecoli0147vs2356 & 0.845 & 0.845 \\
\hline Led7digit02456789vs1 & 0.908 & 0.908 \\
\hline Glass06vs5 & 0.945 & 0.945 \\
\hline Ecoli01vs5 & 0.861 & 0.838 \\
\hline Glass0146vs2 & 0.740 & 0.740 \\
\hline Ecoli0147vs56 & 0.865 & 0.833 \\
\hline Cleveland0vs4 & 0.751 & 0.751 \\
\hline Ecoli0146vs5 & 0.865 & 0.865 \\
\hline Ecoli4 & 0.864 & 0.864 \\
\hline Yeast1vs7 & 0.690 & 0.590 \\
\hline Shuttle0vs4 & 1.000 & 1.000 \\
\hline Glass4 & 0.935 & 0.904 \\
\hline Page-blocks13vs2 & 0.997 & 0.997 \\
\hline Glass016vs5 & 0.888 & 0.888 \\
\hline Shuttle2vs4 & 0.950 & 0.950 \\
\hline Yeast1458vs7 & 0.649 & 0.625 \\
\hline Glass5 & 0.897 & 0.897 \\
\hline Yeast2vs 8 & 0.773 & 0.773 \\
\hline Yeast4 & 0.869 & 0.864 \\
\hline Yeast1289vs7 & 0.771 & 0.752 \\
\hline Yeast5 & 0.965 & 0.965 \\
\hline Ecoli0137vs26 & 0.848 & 0.848 \\
\hline Yeast6 & 0.892 & 0.826 \\
\hline Total Mean & 0.858 & 0.852 \\
\hline
\end{tabular}


Table 14. FURIA-CS against C4.5, SVM, k-NN and FH-GBML with preprocessing approaches and costsensitive learning.

\begin{tabular}{|c|c|c|c|c|c|c|c|c|c|c|c|c|c|}
\hline & FURIA & \multicolumn{3}{|c|}{$\mathrm{C} 45$} & \multicolumn{3}{|c|}{ SVM } & \multicolumn{3}{|c|}{$\mathrm{k}-\mathrm{NN}$} & \multicolumn{3}{|c|}{ FH-GBML } \\
\hline Dataset & CS & SMO & $\mathrm{SMO}+\mathrm{EN}$ & $\mathrm{CS}$ & SMO & $\mathrm{SMO}+\mathrm{EN}$ & $\mathrm{CS}$ & SMO & $\mathrm{SMO}+\mathrm{EN}$ & $\mathrm{CS}$ & SMO & $\mathrm{SMO}+\mathrm{EN}$ & $\mathrm{CS}$ \\
\hline Glass1 & 0.780 & 0.736 & 0.692 & 0.716 & 0.617 & 0.639 & 0.626 & 0.780 & 0.776 & 0.746 & 0.731 & 0.733 & 0.741 \\
\hline EcoliOvs1 & 0.986 & 0.972 & 0.983 & 0.983 & 0.979 & 0.977 & 0.967 & 0.500 & 0.500 & 0.500 & 0.962 & 0.953 & 0.976 \\
\hline Wisconsin & 0.978 & 0.953 & 0.957 & 0.963 & 0.972 & 0.969 & 0.971 & 0.969 & 0.972 & 0.965 & 0.963 & 0.972 & 0.978 \\
\hline Pima & 0.736 & 0.724 & 0.740 & 0.712 & 0.735 & 0.730 & 0.728 & 0.686 & 0.709 & 0.670 & 0.738 & 0.706 & 0.727 \\
\hline Iris0 & 1.000 & 0.990 & 0.990 & 0.990 & 1.000 & 1.000 & 1.000 & 1.000 & 1.000 & 1.000 & 1.000 & 1.000 & 1.000 \\
\hline Glass0 & 0.836 & 0.775 & 0.799 & 0.821 & 0.737 & 0.724 & 0.507 & 0.818 & 0.836 & 0.777 & 0.754 & 0.790 & 0.770 \\
\hline Yeast 1 & 0.707 & 0.709 & 0.695 & 0.677 & 0.710 & 0.706 & 0.674 & 0.677 & 0.707 & 0.685 & 0.700 & 0.704 & 0.701 \\
\hline Vehicle1 & 0.770 & 0.730 & 0.754 & 0.701 & 0.774 & 0.805 & 0.754 & 0.698 & 0.775 & 0.746 & 0.710 & 0.704 & 0.698 \\
\hline Vehicle2 & 0.977 & 0.949 & 0.941 & 0.943 & 0.960 & 0.957 & 0.657 & 0.969 & 0.962 & 0.954 & 0.871 & 0.869 & 0.873 \\
\hline Vehicle3 & 0.785 & 0.728 & 0.740 & 0.728 & 0.761 & 0.788 & 0.790 & 0.708 & 0.763 & 0.735 & 0.712 & 0.727 & 0.694 \\
\hline Haberman & 0.687 & 0.616 & 0.588 & 0.575 & 0.634 & 0.633 & 0.538 & 0.563 & 0.576 & 0.651 & 0.613 & 0.606 & 0.606 \\
\hline Glass0123vs 456 & 0.903 & 0.923 & 0.924 & 0.877 & 0.905 & 0.898 & 0.844 & 0.916 & 0.933 & 0.933 & 0.930 & 0.943 & 0.915 \\
\hline Vehicle0 & 0.946 & 0.918 & 0.907 & 0.928 & 0.963 & 0.961 & 0.949 & 0.947 & 0.941 & 0.946 & 0.893 & 0.869 & 0.887 \\
\hline Ecoli1 & 0.886 & 0.910 & 0.892 & 0.911 & 0.906 & 0.902 & 0.906 & 0.808 & 0.808 & 0.803 & 0.876 & 0.870 & 0.865 \\
\hline New-thyroid2 & 0.951 & 0.965 & 0.977 & 0.980 & 0.991 & 0.988 & 0.982 & 0.988 & 0.986 & 0.991 & 0.980 & 0.977 & 0.951 \\
\hline New-thyroid 1 & 0.963 & 0.963 & 0.988 & 0.974 & 0.994 & 0.986 & 0.968 & 0.988 & 0.986 & 0.991 & 0.951 & 0.991 & 0.965 \\
\hline Ecoli2 & 0.917 & 0.881 & 0.897 & 0.890 & 0.906 & 0.905 & 0.500 & 0.838 & 0.827 & 0.827 & 0.886 & 0.936 & 0.897 \\
\hline Segmento & 0.992 & 0.992 & 0.991 & 0.991 & 0.995 & 0.996 & 0.996 & 0.500 & 0.500 & 0.500 & 0.977 & 0.974 & 0.980 \\
\hline Glass6 & 0.908 & 0.884 & 0.920 & 0.889 & 0.906 & 0.900 & 0.872 & 0.941 & 0.933 & 0.941 & 0.882 & 0.829 & 0.838 \\
\hline Yeast3 & 0.921 & 0.890 & 0.923 & 0.911 & 0.891 & 0.906 & 0.895 & 0.868 & 0.863 & 0.877 & 0.929 & 0.916 & 0.907 \\
\hline Ecoli3 & 0.855 & 0.812 & 0.870 & 0.832 & 0.898 & 0.881 & 0.792 & 0.728 & 0.777 & 0.750 & 0.884 & 0.878 & 0.886 \\
\hline Page-blocks0 & 0.942 & 0.950 & 0.942 & 0.945 & 0.925 & 0.927 & 0.925 & 0.932 & 0.931 & 0.937 & 0.893 & 0.893 & 0.894 \\
\hline Mean & 0.883 & 0.862 & 0.869 & 0.861 & 0.871 & 0.872 & 0.811 & 0.810 & 0.821 & 0.815 & 0.856 & 0.856 & 0.852 \\
\hline$\overline{E c 0}$ & 0.891 & 0.900 & 0.880 & 0.925 & 0.888 & 886 & 0.863 & 0.822 & 0.822 & 0.836 & 0.894 & 0.844 & 0.912 \\
\hline Yeast2vs4v & 0.895 & 0.858 & 0.904 & 0.886 & 0.889 & 0.888 & 0.500 & 0.807 & 0.807 & 0.793 & 0.907 & 0.897 & 0.893 \\
\hline Ecoli067vs35 & 0.872 & 0.850 & 0.812 & 0.882 & 0.832 & 0.835 & 0.802 & 0.820 & 0.815 & 0.855 & 0.812 & 0.875 & 0.818 \\
\hline Ecoli0234vs5 & 0.880 & 0.897 & 0.894 & 0.833 & 0.889 & 0.889 & 0.841 & 0.853 & 0.853 & 0.861 & 0.857 & 0.843 & 0.805 \\
\hline Glass015vs2 & 0.615 & 0.677 & 0.795 & 0.600 & 0.509 & 0.519 & 0.500 & 0.675 & 0.693 & 0.709 & 0.600 & 0.720 & 0.648 \\
\hline Yeast0359vs78 & 0.715 & 0.704 & 0.702 & 0.676 & 0.745 & 0.745 & 0.500 & 0.724 & 0.720 & 0.692 & 0.722 & 0.735 & 0.757 \\
\hline Yeast02579vs368 & 0.915 & 0.914 & 0.913 & 0.899 & 0.901 & 0.906 & 0.500 & 0.902 & 0.901 & 0.898 & 0.909 & 0.893 & 0.900 \\
\hline Yeast0256vs3789 & 0.792 & 0.795 & 0.781 & 0.784 & 0.794 & 0.801 & 0.500 & 0.772 & 0.765 & 0.791 & 0.785 & 0.794 & 0.794 \\
\hline Ecoli046vs5 & 0.889 & 0.870 & 0.886 & 0.831 & 0.886 & 0.886 & 0.869 & 0.928 & 0.928 & 0.936 & 0.832 & 0.806 & 0.966 \\
\hline Ecoli01vs235 & 0.825 & 0.837 & 0.833 & 0.764 & 0.850 & 0.855 & 0.780 & 0.793 & 0.793 & 0.785 & 0.807 & 0.848 & 0.795 \\
\hline Ecoli0267vs35 & 0.827 & 0.815 & 0.817 & 0.852 & 0.825 & 0.853 & 0.785 & 0.840 & 0.832 & 0.802 & 0.833 & 0.799 & 0.831 \\
\hline Glass04vs5 & 0.994 & 0.981 & 0.975 & 0.994 & 0.956 & 0.950 & 0.900 & 0.963 & 0.951 & 0.994 & 0.967 & 0.857 & 0.919 \\
\hline Ecoli0346vs5 & 0.897 & 0.898 & 0.898 & 0.850 & 0.892 & 0.892 & 0.894 & 0.916 & 0.816 & 0.841 & 0.833 & 0.914 & 0.891 \\
\hline Ecoli0347vs56 & 0.769 & 0.856 & 0.854 & 0.758 & 0.908 & 0.906 & 0.813 & 0.792 & 0.500 & 0.836 & 0.860 & 0.852 & 0.832 \\
\hline Yeast05679vs4 & 0.801 & 0.760 & 0.780 & 0.724 & 0.807 & 0.787 & 0.500 & 0.744 & 0.768 & 0.796 & 0.806 & 0.731 & 0.770 \\
\hline Ecoli067vs5 & 0.865 & 0.847 & 0.845 & 0.882 & 0.847 & 0.807 & 0.745 & 0.837 & 0.825 & 0.867 & 0.833 & 0.875 & 0.861 \\
\hline Vowel0 & 0.966 & 0.950 & 0.945 & 0.942 & 0.962 & 0.962 & 0.846 & 0.999 & 0.999 & 0.999 & 0.956 & 0.913 & 0.939 \\
\hline Glass016vs2 & 0.635 & 0.606 & 0.638 & 0.615 & 0.533 & 0.526 & 0.500 & 0.716 & 0.644 & 0.789 & 0.634 & 0.689 & 0.663 \\
\hline Glass2 & 0.738 & 0.639 & 0.745 & 0.641 & 0.615 & 0.690 & 0.595 & 0.716 & 0.771 & 0.695 & 0.677 & 0.599 & 0.709 \\
\hline Ecoli0147vs2356 & 0.845 & 0.827 & 0.822 & 0.877 & 0.882 & 0.872 & 0.726 & 0.759 & 0.795 & 0.827 & 0.850 & 0.845 & 0.862 \\
\hline Led7digit $02456789 \mathrm{vs} 1$ & 0.908 & 0.890 & 0.837 & 0.843 & 0.885 & 0.889 & 0.500 & 0.821 & 0.845 & 0.829 & 0.883 & 0.890 & 0.874 \\
\hline Mean & 0.835 & 0.827 & 0.836 & 0.812 & 0.824 & 0.826 & 0.689 & 0.819 & 0.802 & 0.830 & 0.822 & 0.820 & 0.830 \\
\hline Glass06v & 0.945 & 0.914 & 0.964 & 0.995 & 0.943 & 0.943 & 0.650 & 0.984 & 0.984 & 1.000 & 0.932 & 0.892 & 0.910 \\
\hline Ecoli01vs5 & 0.861 & 0.797 & 0.825 & 0.818 & 0.836 & 0.836 & 0.790 & 0.902 & 0.902 & 0.913 & 0.898 & 0.886 & 0.843 \\
\hline Glass0146vs2 & 0.740 & 0.784 & 0.709 & 0.679 & 0.612 & 0.631 & 0.500 & 0.701 & 0.701 & 0.756 & 0.706 & 0.634 & 0.761 \\
\hline Ecoli0147vs56 & 0.865 & 0.859 & 0.842 & 0.853 & 0.861 & 0.854 & 0.796 & 0.913 & 0.902 & 0.918 & 0.804 & 0.860 & 0.895 \\
\hline Cleveland0vs 4 & 0.751 & 0.790 & 0.760 & 0.689 & 0.878 & 0.914 & 0.748 & 0.834 & 0.834 & 0.858 & 0.752 & 0.705 & 0.686 \\
\hline Ecoli0146vs5 & 0.865 & 0.898 & 0.898 & 0.838 & 0.890 & 0.880 & 0.792 & 0.901 & 0.900 & 0.913 & 0.920 & 0.875 & 0.852 \\
\hline Ecoli4 & 0.864 & 0.779 & 0.904 & 0.863 & 0.920 & 0.920 & 0.952 & 0.842 & 0.810 & 0.818 & 0.930 & 0.929 & 0.942 \\
\hline Yeast1vs7 & 0.609 & 0.700 & 0.737 & 0.613 & 0.786 & 0.774 & 0.500 & 0.739 & 0.699 & 0.745 & 0.719 & 0.642 & 0.738 \\
\hline Shuttle0vs4 & 1.000 & 0.999 & 0.999 & 0.999 & 0.996 & 1.000 & 1.000 & 0.996 & 0.996 & 0.996 & 0.998 & 1.000 & 0.992 \\
\hline Glass4 & 0.935 & 0.886 & 0.865 & 0.843 & 0.957 & 0.910 & 0.912 & 0.891 & 0.915 & 0.886 & 0.886 & 0.961 & 0.874 \\
\hline Page-blocks13vs2 & 0.997 & 0.995 & 0.991 & 0.978 & 0.956 & 0.964 & 0.856 & 0.997 & 0.998 & 0.997 & 0.951 & 0.945 & 0.974 \\
\hline Glass016vs5 & 0.888 & 0.812 & 0.862 & 0.988 & 0.942 & 0.945 & 0.500 & 0.927 & 0.918 & 0.985 & 0.899 & 0.892 & 0.819 \\
\hline Shuttle2vs 4 & 0.950 & 0.991 & 1.000 & 1.000 & 1.000 & 1.000 & 1.000 & 1.000 & 1.000 & 0.950 & 0.994 & 0.987 & 1.000 \\
\hline Yeast1458vs7 & 0.649 & 0.536 & 0.556 & 0.554 & 0.637 & 0.626 & 0.500 & 0.694 & 0.692 & 0.660 & 0.628 & 0.659 & 0.631 \\
\hline Glass5 & 0.897 & 0.880 & 0.775 & 0.942 & 0.951 & 0.941 & 0.973 & 0.937 & 0.973 & 0.932 & 0.767 & 0.797 & 0.884 \\
\hline Yeast2vs8 & 0.773 & 0.833 & 0.819 & 0.865 & 0.766 & 0.764 & 0.766 & 0.720 & 0.737 & 0.801 & 0.744 & 0.722 & 0.741 \\
\hline Yeast 4 & 0.869 & 0.712 & 0.725 & 0.722 & 0.824 & 0.828 & 0.815 & 0.744 & 0.757 & 0.748 & 0.813 & 0.794 & 0.822 \\
\hline Yeast1289vs7 & 0.771 & 0.683 & 0.633 & 0.676 & 0.719 & 0.707 & 0.500 & 0.658 & 0.676 & 0.646 & 0.723 & 0.717 & 0.639 \\
\hline Yeast5 & 0.965 & 0.933 & 0.940 & 0.933 & 0.965 & 0.962 & 0.965 & 0.950 & 0.956 & 0.942 & 0.946 & 0.977 & 0.974 \\
\hline Ecoli0137vs26 & 0.848 & 0.813 & 0.813 & 0.828 & 0.799 & 0.804 & 0.850 & 0.769 & 0.500 & 0.780 & 0.823 & 0.820 & 0.789 \\
\hline Yeast6 & 0.892 & 0.829 & 0.827 & 0.808 & 0.873 & 0.869 & 0.875 & 0.844 & 0.854 & 0.836 & 0.864 & 0.859 & 0.842 \\
\hline Mean & 0.854 & 0.830 & 0.831 & 0.833 & 0.862 & 0.861 & 0.773 & 0.854 & 0.843 & 0.861 & 0.843 & 0.836 & 0.838 \\
\hline Total Mean & 0.858 & 0.840 & 0.845 & 0.835 & 0.853 & 0.853 & 0.758 & 0.840 & 0.838 & 0.841 & 0.828 & 0.822 & 0.835 \\
\hline
\end{tabular}


FURIA with/without preprocessing approaches and cost-sensitive

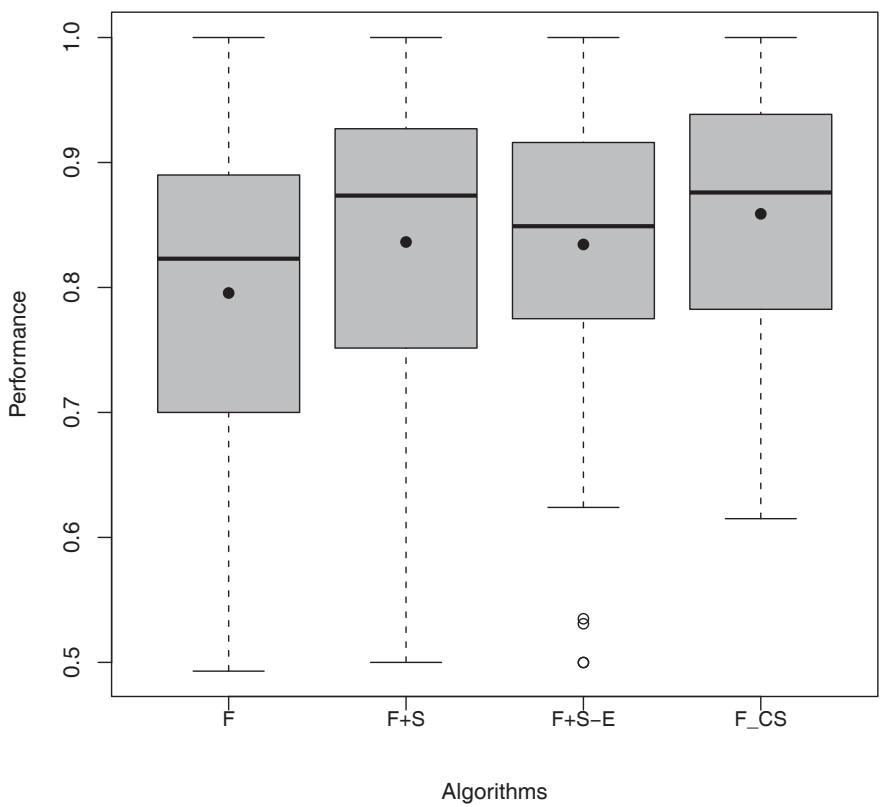

Fig. 1. Dispersion of the performances of FURIA, FURIA+SMOTE, FURIA+SMOTE+ENN and FURIA_CS.

The main conclusions that can be drawn from the preceding table are:

- FURIA_CS with respect to C45+SMOTE, C45+SMOTE+ENN and C45_CS: The performance of FURIA_CS is better than that of C4.5 with both preprocessing methods, and also better than cost-based C4.5. The dispersion of the results is shown in Fig. 2. The $p$-values of the paired comparisons (Wilcoxon test, see Table 15) indicate that the mean performance of FURIA_CS is significantly better than the alternatives.

- FURIA_CS with respect to SVM+SMOTE, SVM+SMOTE+ENN and SVM_CS: The combination of preprocessing techniques and the errorbased versions of SVM improves the results of cost-based SVM (see Fig. 3). Differences between performances of SVM + SMOTE, SVM + SMOTE + ENN and FURIA_CS are not statistically significant, as also shown in Fig. 3. In Table 16 the $p$-values of the paired tests of the best ranked algorithm are shown: hypotheses of equal performance are not rejected for SVM + SMOTE and SVM + SMOTE + ENN, while they are rejected for SVM_CS.

- FURIA_CS with respect to k-NN+SMOTE, k-NN+SMOTE+ENN and k-NN_CS: FURIA_CS is significantly better than k-NN with preprocessing, but the advantage over k-NN_CS is not significant at 95\% confidence level (it would be significant at $92.5 \%$ level). See Fig. 4 and Table 17 for these results. 
FURIA_CS against C4.5+SMOTE, C4.5+SMOTE-ENN and C4.5_CS

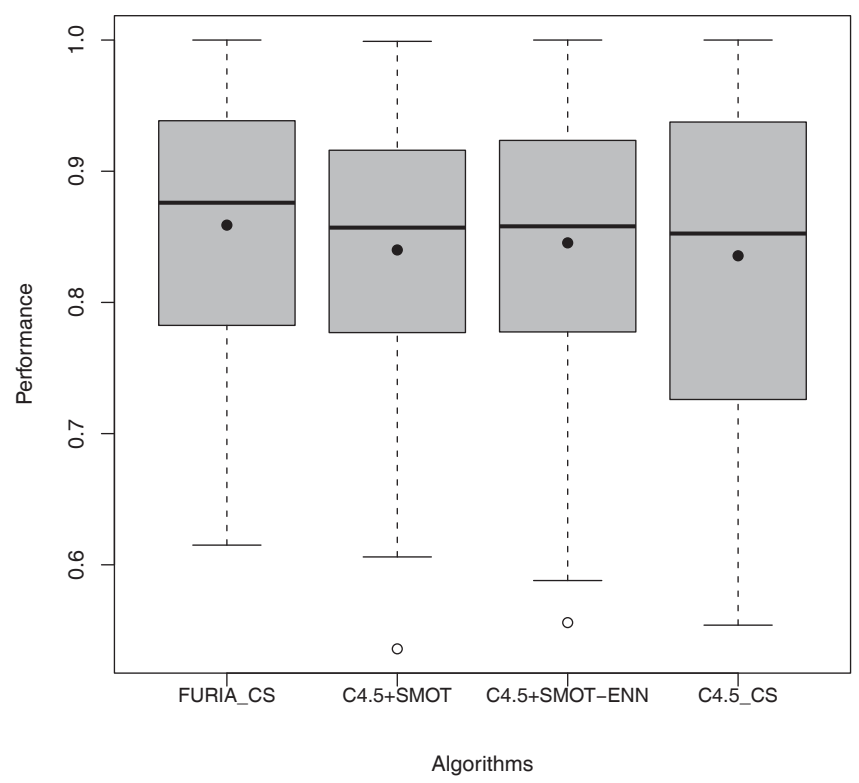

Fig. 2. Performance of FURIA_CS and C4.5 with preprocessing approaches and cost-sensitive learning.

FURIA_CS against SVM+SMOTE, SVM+SMOTE-ENN and SVM_CS

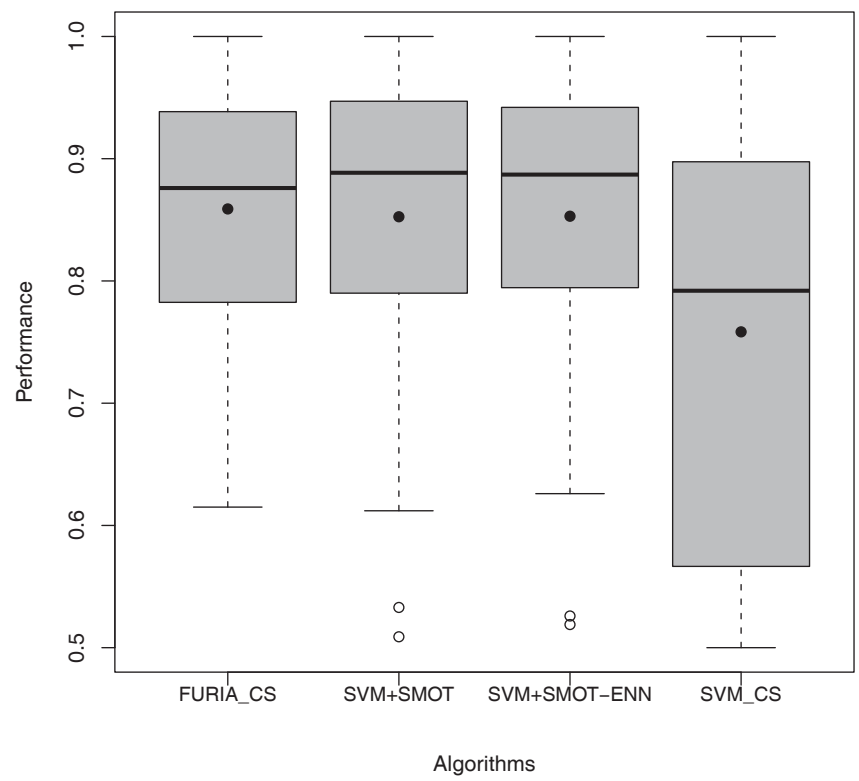

Fig. 3. Performance of FURIA_CS and SVM with preprocessing approaches and cost-sensitive learning. 
Table 15. Wilcoxon's test to compare FURIA_CS against C4.5 with preprocessing approaches and cost-sensitive learning.

\begin{tabular}{lll}
\hline \multicolumn{1}{c}{ Comparison } & $p$-value & Hypothesis $(\alpha=0.05)$ \\
\hline FURIA_CS vs. C4.5 + SMOTE & 0.00044 & Reject \\
FURIA_CS vs. C4.5 + SMOTE + ENN & 0.013 & Reject \\
FURIA_CS vs. C4.5_CS & 0.00012 & Reject \\
\hline
\end{tabular}

Table 16. Wilcoxon's test to compare FURIA_CS against SVM with preprocessing approaches and cost-sensitive learning.

\begin{tabular}{lcc}
\hline \multicolumn{1}{c}{ Comparison } & $p$-value & Hypothesis $(\alpha=0.05)$ \\
\hline FURIA_CS vs. SVM + SMOTE & 0.26 & Not reject \\
FURIA_CS vs. SVM + SMOTE + ENN & 0.20 & Not reject \\
FURIA_CS vs. SVM_CS & $\approx 0$ & Reject \\
\hline
\end{tabular}

Table 17. Wilcoxon's test to compare FURIA_CS against k-NN with preprocessing approaches and cost-sensitive learning.

\begin{tabular}{lcc}
\hline \multicolumn{1}{c}{ Comparison } & $p$-value & Hypothesis $(\alpha=0.05)$ \\
\hline FURIA_CS vs. k-NN + SMOTE & 0.015 & Reject \\
FURIA_CS vs. k-NN + SMOTE+ENN & 0.011 & Reject \\
FURIA_CS vs. k-NN_CS & 0.073 & Not reject \\
\hline
\end{tabular}

Table 18. Wilcoxon's test to compare FURIA_CS against FH-GBML with preprocessing approaches and cost-sensitive learning.

\begin{tabular}{lll}
\hline \multicolumn{1}{c}{ Comparison } & $p$-value & Hypothesis $(\alpha=0.05)$ \\
\hline FURIA_CS vs. FH-GBML + SMOTE & 0.00028 & Reject \\
FURIA_CS vs. FH-GBML + SMOTE + ENN & 0.0016 & Reject \\
FURIA_CS vs. FH-GBML_CS & 0.0014 & Reject \\
\hline
\end{tabular}

- FURIA_CS with respect to FH-GBML + SMOTE, FH-GBML + SMOTE + ENN, and FH-GBML_CS: The performance of FURIA_CS is better than that of FH-GBML both with preprocessing and cost-sensitive learning, as shown in Fig. 5 and Table 18. 
FURIA_CS against k-NN+SMOTE, k-NN+SMOTE-ENN and k-NN_CS

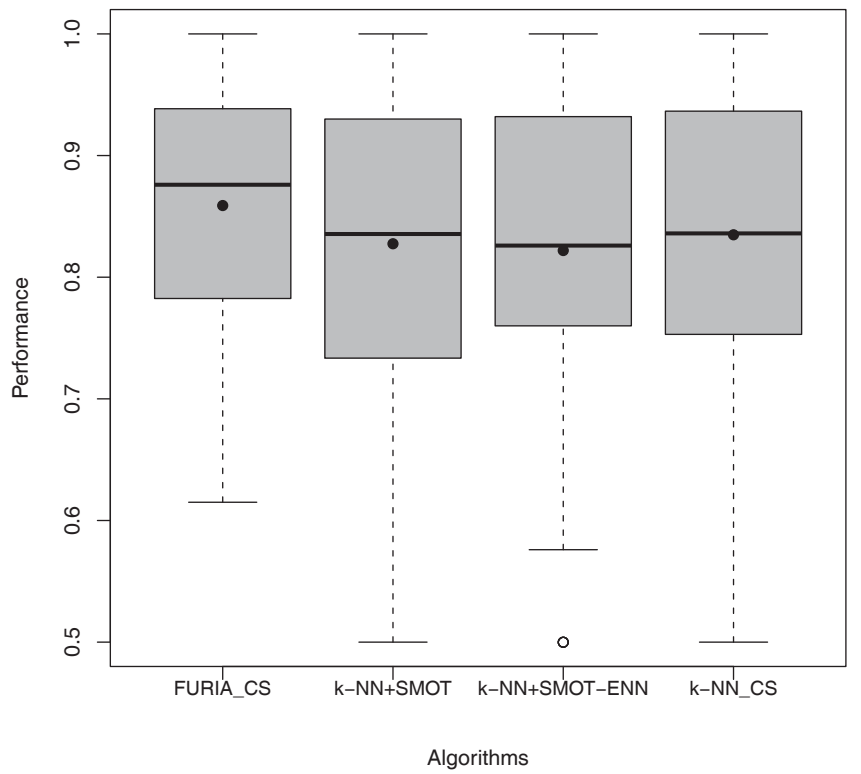

Fig. 4. Performance of FURIA_CS and k-NN with preprocessing approaches and cost-sensitive learning.

FURIA_CS against FH-GBML+preprocessing and FH-GBML_CS

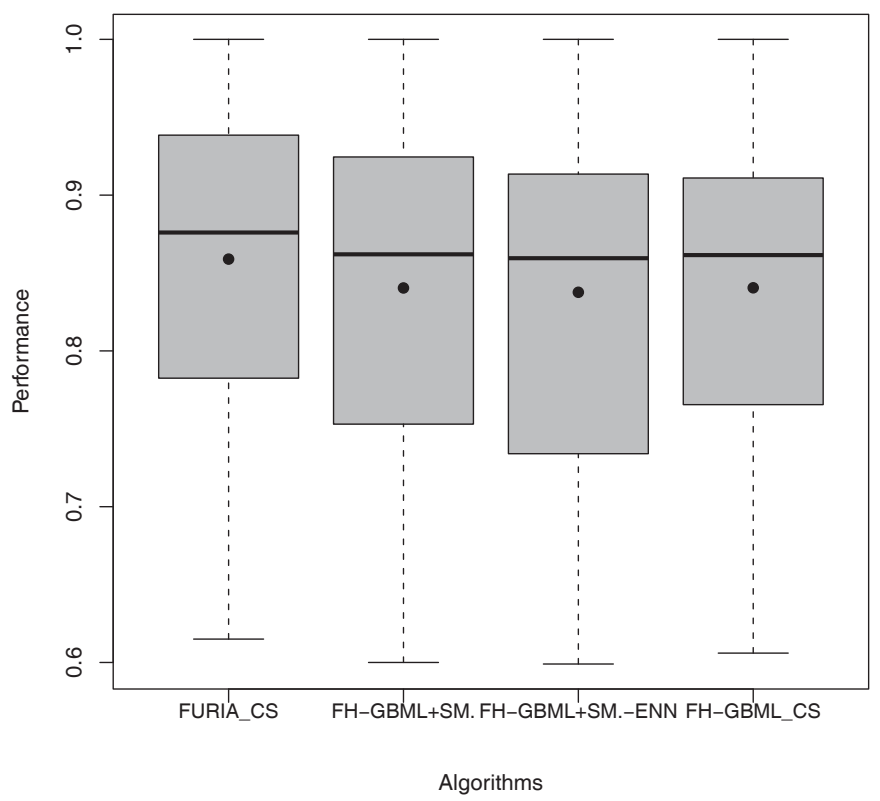

Fig. 5. Performance of FURIA_CS and FH-GBML with preprocessing approaches and costsensitive learning. 


\section{Concluding Remarks}

According to recent literature, ${ }^{32}$ external approaches are expected to perform better than cost-sensitive learning algorithms when tackling imbalanced classification problems. This paper was mainly intended to show that this conclusion must be nuanced. On the one hand, the choice of the learning algorithm is important. In this study, FURIA was chosen because it is very competitive with other fuzzy rule learning algorithms in terms of accuracy. Among other reasons, the accuracy of FURIA is not restricted by the choice of a linguistic partition, and the antecedents of rules dynamically change when an uncovered query appears. These properties allow for a better accuracy than that of static fuzzy linguistic knowledge bases. However, it is not discarded that an improved balance between understandability and accuracy can be achieved with future cost-sensitive generalizations of other fuzzy rule learning algorithms.

On the other hand, the outcome of a cost-sensitive learning algorithm is strongly influenced by the choice of cost matrix. It is intuitive to use a risk proportional to the imbalance ratio for quantifying errors in the minority class. It is also intuitive that correct classifications have a null risk. Unfortunately, the combination of both is not different than making a uniform reweigh of the minority instances. In other words, this cost matrix is equivalent to a crude resampling that is easily improved by state-of-the-art algorithms like SMOTE. As a consequence of this, comparisons between external and internal approaches for solving imbalanced problems should not only be supported by this last cost structure. Best results may be obtained with counter-intuitive assignments. In this study, it has been shown that adding a small penalty to correct classifications of the majority class noticeably improves the results for both AUC and GM metrics.

\section{Acknowledgements}

This work has been funded by the Spanish Ministry of Economy and Competitiveness (Grant TIN2011-24302) and the Spanish Ministry of Education and Science (Grant TIN2009-07727), including EDRF fundings.

\section{Appendix A. FURIA_CS and Penalizations}

The detailed results of the use of different penalizations for correctly classifying negative examples (from the experiment developed in Sec. 5.2.1) are collected below. 
Table 19. AUC-based comparison between penalization factors $\mathrm{PF}_{1}=1 /(2 \mathrm{IR})$, $\mathrm{PF}_{2}=1-(1 / \mathrm{IR})$ and without penalization factor.

\begin{tabular}{|c|c|c|c|c|}
\hline Dataset & $\begin{array}{l}\text { FURIA } \\
\text { Tst }_{\text {AUC }} \\
\end{array}$ & $\begin{array}{c}\text { FURIA-CS (FP2) } \\
\text { Tst }_{\text {AUC }}\end{array}$ & $\begin{array}{c}\text { FURIA-CS (FP1) } \\
\text { TstAUC }\end{array}$ & $\begin{array}{c}\text { FURIA-CS } \\
\text { Tst AUC }\end{array}$ \\
\hline Glass 1 & 0.704 & 0.780 & 0.771 & 0.745 \\
\hline EcoliOvs1 & 0.986 & 0.986 & 0.986 & 0.986 \\
\hline Wisconsin & 0.960 & 0.972 & 0.978 & 0.974 \\
\hline Pima & 0.672 & 0.733 & 0.736 & 0.753 \\
\hline Iris0 & 1.000 & 1.000 & 1.000 & 1.000 \\
\hline Glass0 & 0.797 & 0.836 & 0.836 & 0.819 \\
\hline Yeast 1 & 0.668 & 0.706 & 0.707 & 0.700 \\
\hline Vehicle1 & 0.650 & 0.770 & 0.770 & 0.749 \\
\hline Vehicle2 & 0.969 & 0.977 & 0.977 & 0.975 \\
\hline Vehicle3 & 0.653 & 0.785 & 0.785 & 0.722 \\
\hline Haberman & 0.577 & 0.687 & 0.674 & 0.668 \\
\hline Glass $0123 v s 456$ & 0.868 & 0.903 & 0.893 & 0.873 \\
\hline Vehicle0 & 0.929 & 0.946 & 0.938 & 0.934 \\
\hline Ecoli1 & 0.840 & 0.886 & 0.872 & 0.868 \\
\hline New-thyroid2 & 0.937 & 0.951 & 0.951 & 0.951 \\
\hline New-thyroid 1 & 0.948 & 0.963 & 0.963 & 0.968 \\
\hline Ecoli2 & 0.856 & 0.917 & 0.866 & 0.874 \\
\hline Segmento & 0.987 & 0.992 & 0.988 & 0.988 \\
\hline Glass6 & 0.841 & 0.908 & 0.908 & 0.891 \\
\hline Yeast3 & 0.877 & 0.921 & 0.919 & 0.889 \\
\hline Ecoli3 & 0.769 & 0.855 & 0.794 & 0.781 \\
\hline Page-blocks0 & 0.929 & 0.942 & 0.942 & 0.935 \\
\hline Mean & 0.837 & 0.880 & 0.875 & 0.866 \\
\hline Ecoli034vs5 & 0.819 & 0.891 & 0.883 & 0.866 \\
\hline Yeast2vs4v & 0.825 & 0.895 & 0.884 & 0.859 \\
\hline Ecoli067vs35 & 0.877 & 0.872 & 0.872 & 0.850 \\
\hline Ecoli0234vs5 & 0.838 & 0.880 & 0.866 & 0.866 \\
\hline Glass015vs2 & 0.526 & 0.615 & 0.615 & 0.618 \\
\hline Yeast0359vs78 & 0.584 & 0.715 & 0.629 & 0.662 \\
\hline Yeast02579vs368 & 0.895 & 0.915 & 0.911 & 0.884 \\
\hline Yeast0256vs3789 & 0.688 & 0.792 & 0.774 & 0.713 \\
\hline Ecoli046vs5 & 0.816 & 0.881 & 0.889 & 0.841 \\
\hline Ecoli01vs235 & 0.735 & 0.825 & 0.771 & 0.753 \\
\hline Ecoli0267vs35 & 0.802 & 0.802 & 0.827 & 0.822 \\
\hline Glass04vs5 & 0.994 & 0.994 & 0.994 & 0.994 \\
\hline Ecoli0346vs5 & 0.841 & 0.897 & 0.897 & 0.894 \\
\hline Ecoli0347vs56 & 0.815 & 0.796 & 0.769 & 0.751 \\
\hline Yeast05679vs4 & 0.696 & 0.782 & 0.801 & 0.709 \\
\hline Ecoli067vs5 & 0.840 & 0.855 & 0.865 & 0.862 \\
\hline Vowel0 & 0.950 & 0.966 & 0.963 & 0.954 \\
\hline Glass016vs2 & 0.519 & 0.628 & 0.635 & 0.565 \\
\hline Glass2 & 0.558 & 0.738 & 0.717 & 0.690 \\
\hline Ecoli0147vs2356 & 0.821 & 0.845 & 0.825 & 0.805 \\
\hline Led7digit02456789vs 1 & 0.881 & 0.908 & 0.908 & 0.894 \\
\hline Mean & 0.776 & 0.832 & 0.823 & 0.802 \\
\hline Glass06vs5 & 0.945 & 0.945 & 0.945 & 0.945 \\
\hline Ecoli01vs5 & 0.838 & 0.838 & 0.861 & 0.836 \\
\hline Glass0146vs2 & 0.497 & 0.740 & 0.658 & 0.634 \\
\hline Ecoli0147vs56 & 0.796 & 0.858 & 0.865 & 0.830 \\
\hline Cleveland0vs4 & 0.748 & 0.751 & 0.751 & 0.754 \\
\hline Ecoli0146vs5 & 0.744 & 0.865 & 0.834 & 0.813 \\
\hline Ecoli4 & 0.815 & 0.864 & 0.840 & 0.840 \\
\hline Yeast1vs7 & 0.546 & 0.690 & 0.609 & 0.580 \\
\hline Shuttle0vs 4 & 1.000 & 1.000 & 1.000 & 1.000 \\
\hline Glass 4 & 0.870 & 0.904 & 0.935 & 0.870 \\
\hline Page-blocks13vs2 & 0.997 & 0.997 & 0.997 & 0.997 \\
\hline Glass016vs5 & 0.844 & 0.844 & 0.888 & 0.844 \\
\hline Shuttle2vs4 & 0.950 & 0.950 & 0.950 & 0.950 \\
\hline Yeast1458vs7 & 0.493 & 0.542 & 0.649 & 0.543 \\
\hline Glass5 & 0.847 & 0.897 & 0.897 & 0.895 \\
\hline Yeast2vs 8 & 0.773 & 0.773 & 0.773 & 0.773 \\
\hline Yeast4 & 0.545 & 0.770 & 0.869 & 0.633 \\
\hline Yeast1289vs7 & 0.566 & 0.599 & 0.771 & 0.530 \\
\hline Yeast5 & 0.885 & 0.965 & 0.914 & 0.905 \\
\hline Ecoli0137vs26 & 0.746 & 0.746 & 0.848 & 0.746 \\
\hline Yeast6 & 0.739 & 0.802 & 0.892 & 0.781 \\
\hline Mean & 0.771 & 0.825 & 0.845 & 0.795 \\
\hline Total Mean & 0.794 & 0.846 & 0.847 & 0.821 \\
\hline
\end{tabular}


Table 20. GM-base comparison between penalization factors $\mathrm{PF}_{1}=1 /(2 \mathrm{IR})$, $\mathrm{PF}_{2}=1-(1 / \mathrm{IR})$ and without penalization factor.

\begin{tabular}{|c|c|c|c|c|}
\hline Dataset & $\begin{array}{l}\text { FURIA } \\
\text { Tst }_{\text {GM }}\end{array}$ & $\begin{array}{c}\text { FURIA-CS (FP2) } \\
\text { Tst }_{\mathrm{GM}}\end{array}$ & $\begin{array}{c}\text { FURIA-CS (FP1) } \\
\text { Tst }_{\mathrm{GM}}\end{array}$ & $\begin{array}{c}\text { FURIA-CS } \\
\text { Tst }_{\text {GM }}\end{array}$ \\
\hline Glass1 & 0.794 & 0.774 & 0.760 & 0.731 \\
\hline Ecoli0vs1 & 0.995 & 0.986 & 0.986 & 0.986 \\
\hline Wisconsin & 0.975 & 0.971 & 0.977 & 0.974 \\
\hline Pima & 0.705 & 0.720 & 0.715 & 0.742 \\
\hline Iris0 & 1.000 & 1.000 & 1.000 & 1.000 \\
\hline Glass0 & 0.855 & 0.823 & 0.823 & 0.813 \\
\hline Yeast 1 & 0.678 & 0.682 & 0.682 & 0.676 \\
\hline Vehicle1 & 0.694 & 0.751 & 0.751 & 0.734 \\
\hline Vehicle2 & 0.982 & 0.977 & 0.977 & 0.974 \\
\hline Vehicle3 & 0.758 & 0.766 & 0.766 & 0.692 \\
\hline Haberman & 0.532 & 0.667 & 0.637 & 0.645 \\
\hline Glass0123vs 456 & 0.838 & 0.902 & 0.891 & 0.868 \\
\hline Vehicle0 & 0.925 & 0.946 & 0.936 & 0.933 \\
\hline Ecoli1 & 0.893 & 0.883 & 0.866 & 0.863 \\
\hline New-thyroid2 & 0.924 & 0.950 & 0.950 & 0.950 \\
\hline New-thyroid 1 & 0.957 & 0.962 & 0.962 & 0.967 \\
\hline Ecoli2 & 0.883 & 0.916 & 0.859 & 0.867 \\
\hline Segmento & 0.992 & 0.992 & 0.988 & 0.988 \\
\hline Glass6 & 0.854 & 0.903 & 0.903 & 0.884 \\
\hline Yeast 3 & 0.860 & 0.920 & 0.918 & 0.884 \\
\hline Ecoli3 & 0.699 & 0.850 & 0.765 & 0.749 \\
\hline Page-blocks0 & 0.935 & 0.941 & 0.941 & 0.933 \\
\hline Mean & 0.850 & 0.876 & 0.865 & 0.857 \\
\hline Ecoli034vs5 & 0.816 & 0.882 & 0.872 & 0.839 \\
\hline Yeast2vs4v & 0.865 & 0.891 & 0.875 & 0.850 \\
\hline Ecoli067vs35 & 0.794 & 0.771 & 0.771 & 0.747 \\
\hline Ecoli0234vs5 & 0.761 & 0.870 & 0.854 & 0.854 \\
\hline Glass015vs2 & 0.050 & 0.373 & 0.373 & 0.376 \\
\hline Yeast0359vs78 & 0.557 & 0.679 & 0.493 & 0.583 \\
\hline Yeast $02579 v s 368$ & 0.861 & 0.913 & 0.906 & 0.877 \\
\hline Yeast0256vs 3789 & 0.624 & 0.773 & 0.742 & 0.649 \\
\hline Ecoli046vs5 & 0.852 & 0.870 & 0.878 & 0.822 \\
\hline Ecoli01vs235 & 0.608 & 0.795 & 0.715 & 0.694 \\
\hline Ecoli0267vs35 & 0.738 & 0.773 & 0.805 & 0.797 \\
\hline Glass04vs5 & 0.982 & 0.994 & 0.994 & 0.994 \\
\hline Ecoli0346vs5 & 0.931 & 0.890 & 0.890 & 0.887 \\
\hline Ecoli0347vs56 & 0.737 & 0.771 & 0.729 & 0.699 \\
\hline Yeast05679vs4 & 0.555 & 0.770 & 0.784 & 0.648 \\
\hline Ecoli067vs5 & 0.818 & 0.828 & 0.837 & 0.834 \\
\hline Vowel0 & 0.978 & 0.966 & 0.962 & 0.949 \\
\hline Glass016vs2 & 0.162 & 0.535 & 0.486 & 0.321 \\
\hline Glass2 & 0.475 & 0.731 & 0.587 & 0.554 \\
\hline Ecoli0147vs2356 & 0.912 & 0.825 & 0.802 & 0.773 \\
\hline Led7digit02456789vs1 & 0.948 & 0.902 & 0.902 & 0.886 \\
\hline Mean & 0.711 & 0.800 & 0.773 & 0.744 \\
\hline Glass06vs5 & 0.955 & 0.936 & 0.936 & 0.936 \\
\hline Ecoli01vs5 & 0.763 & 0.820 & 0.845 & 0.818 \\
\hline Glass0146vs2 & 0.340 & 0.707 & 0.511 & 0.471 \\
\hline Ecoli0147vs56 & 0.811 & 0.848 & 0.854 & 0.814 \\
\hline Cleveland0vs 4 & 0.635 & 0.702 & 0.702 & 0.705 \\
\hline Ecoli0146vs5 & 0.779 & 0.856 & 0.801 & 0.704 \\
\hline Ecoli4 & 0.835 & 0.854 & 0.811 & 0.811 \\
\hline Yeast1vs7 & 0.323 & 0.679 & 0.358 & 0.311 \\
\hline Shuttle0vs4 & 1.000 & 1.000 & 1.000 & 1.000 \\
\hline Glass4 & 0.925 & 0.892 & 0.926 & 0.855 \\
\hline Page-blocks13vs2 & 0.993 & 0.997 & 0.997 & 0.997 \\
\hline Glass016vs5 & 0.868 & 0.736 & 0.873 & 0.736 \\
\hline Shuttle2vs 4 & 0.971 & 0.941 & 0.941 & 0.941 \\
\hline Yeast $1458 \mathrm{vs} 7$ & 0.081 & 0.493 & 0.495 & 0.195 \\
\hline Glass5 & 0.899 & 0.797 & 0.797 & 0.795 \\
\hline Yeast 2vs 8 & 0.614 & 0.728 & 0.728 & 0.728 \\
\hline Yeast 4 & 0.506 & 0.763 & 0.866 & 0.455 \\
\hline Yeast1289vs7 & 0.152 & 0.386 & 0.636 & 0.162 \\
\hline Yeast 5 & 0.916 & 0.964 & 0.910 & 0.900 \\
\hline Ecoli0137vs26 & 0.269 & 0.538 & 0.740 & 0.540 \\
\hline Yeast6 & 0.606 & 0.789 & 0.885 & 0.739 \\
\hline Mean & 0.662 & 0.782 & 0.796 & 0.696 \\
\hline Total Mean & 0.741 & 0.819 & 0.809 & 0.765 \\
\hline
\end{tabular}




\section{References}

1. N. Abe, B. Zadrozny and J. Langford, An iterative method for multi-class costsensitive learning, in Proc. 10th ACM SIGKDD Int. Conf. Knowledge Discovery and Data Mining (2004), pp. 3-11.

2. J. Alcalá-Fdez, A. Fernández, J. Luengo, J. Derrac, S. García, L. Sánchez and F. Herrera, KEEL Data-Mining Software Tool: Data set repository, integration of algorithms and experimental analysis framework, J. Multiple-Valued Logic and Soft Computing 17(2-3) (2011) 255-287.

3. G. Batista, R. Prati and M. Monard, A study of the behaviour of several methods for balancing machine learning training data, SIGKDD Explorations 6(1) (2004) 20-29.

4. J. Berger, Statistical Decision Theory and Bayesian Analysis (Springer-Verlag, 1985).

5. N. V. Chawla, K. W. Bowywe, L.O. Hall and W. P. Kegelmeyer, SMOTE: Synthetic minority over-sampling technique, J. Artif. Intell. Res. 16 (2002) 321-357.

6. N. V. Chawla, C4.5 and imbalanced data sets: investigating the effect of sampling method, probabilistic estimate, and decision tree structure, in Proc. ICML Workshop on Class Imbalances (2003).

7. W. Cohen, Fast effective rule induction, in A. Prieditis and S. Russel (eds.), in Proc. 12th Int. Conf. Machine Learning (ICML) (1995), pp. 115-123.

8. T. Cover and P. Hart, Nearest neighbor pattern classification, IEEE Trans. Information Theory 13 (1967) 21-27.

9. K. Crockett, Z. Bandar and J. O'shea, On producing balanced fuzzy decision tree classifiers, in Proc. IEEE Int. Conf. Fuzzy Systems (FUZZIEEE) (2006), pp. 17561762 .

10. A. P. Bradley, The use of the area under the ROC curve in the evaluation of machine learning algorithms, Pattern Recognition 30(7) (1997) 1145-1159.

11. N. V. Chawla, N. Japkowicz and A. Kolcz, Editorial: Special issue on learning from imbalanced data sets, SIGKDD Explorations 6(1) (2004) 1-6.

12. J. Demsar, Statistical comparisons of classifiers over multiple datasets, J. Mach. Learn. Res. 7 (2006) 1-30.

13. J. P. Dmochowski, P. Sajda and L. C. Parra, Maximum likelihood in cost-sensitive learning: Model specification, approximators, and upper bounds, J. Mach. Learn. Res. 11 (2010) 3313-3332.

14. D. P. Metacost, A general method for making classifiers cost-sensitive, in Proc. 5th Int. Conf. Knowledge Discovery and Data Mining (KDD) (1999), pp. 155-164.

15. C. Drummond and R. C. Holte, Exploiting the cost (in)sensitivity of decision tree splitting criteria, in Proc. 17th Int. Conf. Machine Learning (ICML) (2000), pp. 239 146.

16. P. Ducange, B. Lazzerini and F. Marcelloni, Multi-objetive genetic fuzzy classifiers for imbalanced and cost-sensitive datasets, Soft Computing 14(7) (2010), pp. 713-728.

17. C. Elkan, The foundations of cost-sensitive learning, in Proc. 17th IEEE Int. Joint Conf. Artificial Intelligence (IJCAI) (2001), pp. 973-978.

18. A. Fernández, S. García, M. J. del Jesus and F. Herrera, A study of the behaviour of linguistic fuzzy rule based classification systems in the framework of imbalanced data-sets, Fuzzy Sets and Systems 159 (2008) 2378-2398.

19. M. Fazzolari, R. Alcala, Y. Nojima, H. Ishibuchi and F. Herrera, A review of the application of multiobjective evolutionary fuzzy systems: Current status and further directions, IEEE Trans. Fuzzy Systems 21(1) (2013) 45-65.

20. A. Fernández, M. J. del Jesus and F. Herrera, Hierarchical fuzzy rule based classification systems with genetic rule selection for imbalanced data-sets, Int. J. Approximate Reasoning 50 (2009) 561-577. 
21. A. Fernández, M. J. del Jesus and F. Herrera, On the influence of an adaptative inference system in fuzzy rule based classification systems for imbalanced data-sets, Expert Systems with Applications 36 (2009) 9805-9812.

22. A. Fernández, M. J. del Jesus and F. Herrera, On the 2-tuples based genetic tuning performance for fuzzy rule based classification systems in imbalanced data-sets, Information Sciences 180 (2010) 1268-1291.

23. J. Huang and C. Ling, Using auc and accuracy in evaluating learning algorithms - appendices, IEEE Transactions on Knowledge and Data Engineering 17 (2005) 299-310.

24. Y. M. Huang, C. M. Hung and H. Jiau, Evaluation of neural networks and data mining methods on a credit assessment task for class imbalance problem, Nonlinear Analysis: Real World Applications 7(4) (2006) 720-747.

25. J. C. Hühn and E. Hüllermeier, FURIA: an algorithm for unordered fuzzy rule induction, Data Mining and Knowledge Discovery 19 (2009) 293-319.

26. J. C. Hühn and E. Hüllermeier, FURIA: Fuzzy Unordered Rule Induction Algorithm, URL: http://www . uni-marburg.de/fb12/kebi/research/software/furia (2009).

27. J. C. Hühn and E. Hüllermeier, An analysis of the FURIA algorithm for fuzzy rule induction, in Advances in Machine Learning I (2010), pp. 32-344.

28. H. Ishibuchi, T. Yamamoto and T. Nakashima, Hybridization of fuzzy GBML approaches for pattern classification problems, IEEE Trans. System, Man and Cybernetics, Part B 35 (2005) 359-365.

29. N. Japkowicz and S. Stephen, The class imbalance problem: a systematic study, Intelligent Data Analysis 6(5) (2002) 429-450.

30. M. Kubal, R. Holte and S. Matwin, Learning when negative examples abound, in Proc. European Conf. Machine Learning (ECML) (1997), pp. 146-153.

31. M. Kubat and S. Matwin, Addressing the curse of imbalanced training sets: one-sided selection, in Proc. Int. Conf. Machine Learning (ICML) (1997), pp. 170-186.

32. V. Lopez, A. Fernandez, J. G. Moreno-Torres and F. Herrera, Analysis of preprocessing versus cost-sensitive learning for imbalanced classification. Open problems on intrinsic data characteristics, Expert Systems with Applications 39 (2012) 65856608 .

33. D. Margineantu, Methods for cost-sensitive learning, Technical report, Department of Computer Science, Oregon State University, Corvallis, OR, USA (2001).

34. D. Margineantu, Class probability estimation and cost-sensitive classification decisions, in Proc. 13th European Conf. Machine Learning (ECML) (2002), pp. 270281.

35. M. Mazurowski, P. Habas, J. Zurada, J. Lo, J. Baker and G. Tourassi, Training neural network classifiers for medical decision making: The effect of imbalanced datasets on classification performance, Neural Networks 21(2-3) (2008) 417-436.

36. A. Palacios, L. Sánchez and I. Couso, Linguistic cost-sensitive learning of genetic fuzzy classifiers for imprecise data, Int. J. Approximate Reasoning 52 (2011) 841-862.

37. A. Palacios, L. Sánchez and I. Couso, Equalizing imbalanced imprecise datasets for genetic fuzzy classifiers, Int. J. Computational Intelligence Systems: Special Issue on Evolutionary Fuzzy Systems 5(2) (2012) 276-296.

38. X. Peng and I. King, Robust BMPM training based on second-order cone programming and its application in medical diagnosis, Neural Networks 21(2-3) (2008) 450457.

39. C. Phua, D. Alahakoon and V. Lee, Minority report in fraud detection: classification of skewed data, SIGDKK Explorations Newsletter 6(1) (2004) 50-59. 
40. F. Provost and T. Fawcett, Robust classification systems for imprecise environments, in Proc. AAAI (1998), pp. 706-713.

41. J. Quinlan, C4.5: Programs for Machine Learning (Morgan Kaufmann, 1993).

42. V. Soler, J. Cerquides, J. Sabria, J. Roig and M. Prim, Imbalanced datasets classification by fuzzy rule extraction and genetic algorithms, in Proc. IEEE Int. Conf. Data Mining Workshop (2006), pp. 330-336.

43. Y. Sun, M. Kamel, A. K. C. Wong and Y. Wang, Cost-sensitive boosting for classification of imbalanced data, Pattern Recognition 40 (2007) 3358-3378.

44. Y. Sun, A. K. C. Wong and M. Kemel, Classification of imbalanced data: A review, Int. J. Pattern Recognition and Artificial Intelligence 23(4) (2009) 687-719.

45. P. Turney, Types of cost in inductive concept learning, in Proc. Workshop on CostSensitive Learning at the 17th Int. Conf. Machine Learning (WCSL at ICML) (2000), pp. 15-21.

46. V. Vapnik, Statistical Learning Theory (Wiley, New York, USA, 1998).

47. S. Visa and A. Ralescu, Learning imbalanced and overlapping classes using fuzzy sets, in Proc. Int. Conf. Machine Learning - Workshop on Learning from Imbalanced Datasets II (2003).

48. S. Visa and A. Ralescu, The effect of imbalanced data class distribution on fuzzy classifiers-experimental study, in Proc. IEEE Int. Conf. Fuzzy Systems (2005), pp. $749-754$.

49. G. Weiss, Mining with rarity: a unifying framework, SIGKDD Explorations 6(1) (2004) $7-19$.

50. F. Xia, Y. Yan, L. Zhou, F. Li, M. Cai and D. Zeng, A closed-form reduction of multiclass cost-sensitive learning to weighted multi-class learning, Pattern Recognition 42 (2009) 1572-1581.

51. L. Xu, M. Chow and L. Taylor, Power distribution fault cause identification with imbalanced data using the data mining-based fuzzy classification e-algorithm, IEEE Trans. Power Systems 22(1) (2007) 164-171.

52. D. L. Wilson, Asymptotic properties of nearest neighbor rules using edited data, IEEE Trans. Systems, Man and Cybernetics 2 (1972) 408-421.

53. B. Zadrozny and C. Elkan, Learning and making decisions when costs and probabilities are both unknown, in Proc. 7th Int. Conf. Knowledge Discovery and Data Mining (KDD) (2001), pp. 204-213.

54. B. Zadrozny, One-benefit learning: cost-sensitive learning with restricted cost information, in Proc. 1st Int. Workshop on Utility-based Data Mining (2005), pp. 53-58.

55. Z. H. Zhou and X. Y. Liu, On multi-class cost-sensitive learning, in Proc. 21st National Conf. Artificial Intelligence (2006), pp. 567-572.

56. Z. H. Zhou and X. Y. Liu, Training cost-sensitive neural networks with methods addressing the class imbalance problem, IEEE Trans. Knowledge and Data Engineering 18(1) (2006) 63-77. 